\title{
Nucleotide-binding oligomerization domain 1 acts in concert with the cholecystokinin receptor agonist, cerulein, to induce IL-33-dependent chronic pancreatitis
}

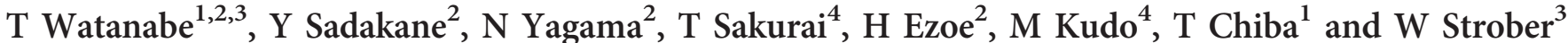

Nucleotide-binding oligomerization domain 1 (NOD1) fulfills important host-defense functions via its responses to a variety of gut pathogens. Recently, however, we showed that in acute pancreatitis caused by administration of cholecystokinin receptor (CCKR) agonist (cerulein) NOD1 also has a role in inflammation via its responses to gut commensal organisms. In the present study, we explored the long-term outcome of such NOD1 responsiveness in a new model of chronic pancreatitis induced by repeated administration of low doses of cerulein in combination with NOD1 ligand. We found that the development of chronic pancreatitis in this model requires intact NOD1 and type I IFN signaling and that such signaling mediates a macrophage-mediated inflammatory response that supports interleukin (IL)-33 production by acinar cells. The IL-33, in turn, has a necessary role in the induction of IL-13 and TGF- $\beta 1$, factors causing the fibrotic reaction characteristic of chronic pancreatitis. Interestingly, the Th2 effects of IL-33 were attenuated by the concomitant type I IFN response since the inflammation was marked by clear increases in IFN- $\gamma$ and TNF- $\alpha$ production but only marginal increases in IL-4 production. These studies establish chronic pancreatitis as an IL-33-dependent inflammation resulting from synergistic interactions between the NOD1 and CCKR signaling pathways.

\section{INTRODUCTION}

Pancreatic inflammatory disease can occur as an acute or a chronic form of inflammation that differ from one another both clinically and pathologically. Acute pancreatitis refers to new-onset pancreatic inflammation occurring in a previously un-inflamed pancreas that may subside prior to causing permanent changes in the pancreas or may cause permanent changes if recurrent; in contrast, chronic pancreatitis refers to on-going pancreatic inflammation of varying severity in a pancreas already reflecting permanent inflammatory changes. ${ }^{1,2}$ In addition, the presence of long-standing inflammation in chronic pancreatitis is not simply due to the continuation of the inflammatory process present in the acute disease. Instead, it results from the activation of new pathological processes that cause the development of inflammation only evident in chronic pancreatitis such as severe pancreatic atrophy and fibrosis. It is these new features of inflammation that cause the insufficiency in both exocrine and endocrine pancreatic function typical of chronic pancreatitis. ${ }^{2,3}$

A well-accepted hypothesis regarding the pathogenesis of both acute and chronic pancreatitis is that pancreatic inflammation is initiated by various environmental factors (such as excessive drinking of alcohol) capable of causing pathologic triggering of the cholecystokinin receptor (CCKR) signaling pathway within pancreatic acinar cells and subsequently, the excessive conversion of trypsinogen to trypsin. ${ }^{4,5}$ This, in turn, leads to autodigestion of pancreatic tissue and the influx of inflammatory cells that cause the pancreatic inflammation. ${ }^{1,2}$ This concept of pancreatitis pathogenesis is fully supported by the fact that mutations in a molecule that

${ }^{1}$ Department of Gastroenterology and Hepatology, Kyoto University Graduate School of Medicine, Kyoto, Kyoto, Japan. ${ }^{2}$ Center for Innovation in Immunoregulative Technology and Therapeutics, Kyoto University Graduate School of Medicine, Kyoto, Kyoto, Japan. ${ }^{3}$ Mucosal Immunity Section, Laboratory of Host Defenses, National Institute of Allergy and Infectious Diseases, National Institutes of Health, Bethesda, Maryland, USA and ${ }^{4}$ Department of Gastroenterology and Hepatology, Kinki University Graduate School of Medicine, Osaka-sayama, Osaka, Japan. Correspondence: T Watanabe (tmhrwtnb@kuhp.kyoto-u.ac.jp). 
inhibits cationic trypsinogen activation, the serine protease inhibitor Kazal type 1 (SPINK1) or mutations in cationic trypsinogen (PRSS1) cause hereditary pancreatitis in humans due to constitutive activation of trypsinogen. ${ }^{6}$ However, autodigestion of acinar cells by activated trypsin may not completely account for development of pancreatitis in all cases since pancreatitis can arise from very diverse factors. ${ }^{7}$ In addition, it has been reported that mice that are genetically deficient in trypsinogen nevertheless exhibit pancreatic and systemic inflammation similar to that in wild-type mice subjected to acute and chronic experimental cerulein-induced pancreatitis. ${ }^{8,9}$ Given the fact that cerulein, a CCKR agonist, is a strong inducer of trypsinogen activation, ${ }^{10}$ these studies thus alert us to the possibility that excessive activation of trypsinogen is not the only mechanism contributing to the development of pancreatitis. ${ }^{11}$

In line with this idea, evidence has recently emerged that factors relating to innate immune responses activated by microbe-associated molecular patterns (MAMPs) ${ }^{12,13}$ and damage-associated molecular patterns (DAMPs) ${ }^{14}$ also contribute to the development of pancreatitis. This was first suggested by the fact that bacterial colonization of the inflamed pancreas occurs in severe forms of acute pancreatitis characterized by local and extrapancreatic complications and, in fact, infection of necrotic pancreatic tissue is one of the most important causes of mortality in acute pancreatitis. ${ }^{1}$ The relationship between bacterial colonization and the severity of the pancreatitis is also supported by studies showing that bowel sterilization via antibiotic treatment reduces or prevents pancreatic inflammation, infection, and mortality in various experimental pancreatitis models. ${ }^{15,16}$ In addition, DAMPs released from autodigested pancreatic tissue have been shown to amplify the inflammation through the activation of innate immune receptors. ${ }^{17,18}$

In studies further supporting the role of innate factors in the pathogenesis of pancreatitis we have previously shown that nucleotide-binding oligomerization domain 1 (NOD1), an intracellular innate immune receptor that detects small peptide components derived from bacterial wall peptidoglycan may also contributes to the development of pancreatitis. ${ }^{15}$ In particular, we showed that NOD1 signaling arising from the detection of intestinal microflora by pancreatic acinar cells is necessary for cerulein-induced acute pancreatitis. ${ }^{15}$ Moreover, we established a novel model of acute pancreatitis that is induced by the synergistic activity of low dose of cerulein (that does not itself induce pancreatitis) and FK156, an activator of NOD1 that mimics the effect of NOD1 ligand expression by gut bacteria breaching the mucosal barrier. ${ }^{15}$ Whereas these previous studies established the importance of NOD1-mediated innate immune responses in acute pancreatitis, they did not provide information about the role of these responses in the chronic form of this disease. To address this question we created a model of chronic pancreatitis induced by repeated administration of low-dose cerulein and NOD1 ligand (FK565) and have used this model to explore the immune mechanisms driving chronic pancreatitis. We showed that in this model, chronic activation of NOD1 in pancreatic acinar cells gives rise to a fibroinflammatory disorder of the pancreas mediated by innate interleukin (IL)-33 and adaptive IL-13 responses.

\section{RESULTS}

\section{Repeated injection of low-dose cerulein together with NOD1 ligand (FK565) induces chronic pancreatitis}

In a previous study, we established a new model of acute pancreatitis that is induced by administration of low doses of the CCKR agonist, cerulein, together with a single dose of NOD1 ligand. ${ }^{15}$ Neither of these agents was capable of causing pancreatitis when administered alone and it was thus clear that the induction of pancreatitis required their synergistic interaction. To better analyze the consequences of this interaction we developed a model of chronic pancreatitis in which C57BL/6 mice were subjected to combination cerulein/FK565 treatment administered twice a week for 7 weeks; this combination treatment consisted of an intraperitoneal (IP) injection of FK565 (NOD1 ligand; $50 \mu \mathrm{g}$ ) and three hourly IP injections of a low dose of cerulein $\left(20 \mu \mathrm{g} \mathrm{kg}^{-1}\right)$. In the studies below we refer to this method of inducing chronic pancreatitis as the FK565-cerulein CP regimen.

As shown in Figure 1a,b, the effect of administration of the FK565-cerulein CP regimen differed markedly from the effects of administration of FK565 alone or cerulein alone (administered at the same dose as in the administration of the FK565-cerulein CP regimen). Thus, as shown in Figure 1a and Supplementary Figure S1a online, mice subjected to repeated administration of FK565 alone did not exhibit elevated serum levels of amylase or a positive chronic pancreatitis pathology score, whereas mice subjected to repeated administration of cerulein alone exhibited mild pancreatitis as indicated by the presence of increased serum amylase levels and increased pathology scores; evidently, even low-dose cerulein can cause pancreatitis when administered repeatedly. A different picture was obtained with administration of the FK565-cerulein CP regiment since in this case the mice exhibited full-blown chronic pancreatitis as indicated by the presence of increased serum amylase levels, decreased pancreatic weight and a very high pathology score. The fact that serum amylase levels were not as high as in mice with mild pancreatitis induced by administration of cerulein alone reflects the fact that administration of the FK565-cerulein $\mathrm{CP}$ regimen causes pancreatic atrophy and associated fibrosis (see below).

As shown in Figure 1b, the above findings were corroborated by $\mathrm{H} \& \mathrm{E}$ staining and other tissue staining studies described below and previously. ${ }^{19}$ With respect to H\&E staining, repeated administration of FK565 caused no changes in pancreatic architecture, whereas repeated administration of cerulein alone led to decreased acinar cell adhesion and expanded peri-acinar extracellular spaces containing a low number of immune cells. In contrast, mice administered the FK565-cerulein $\mathrm{CP}$ regimen exhibited extensive loss of normal acinar architecture, acinar cell loss and a large increase in infiltrating immune cells. These findings fit nicely with tissue staining studies to detect the 
a

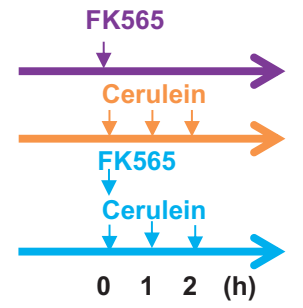

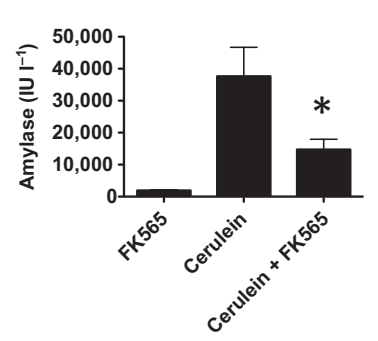
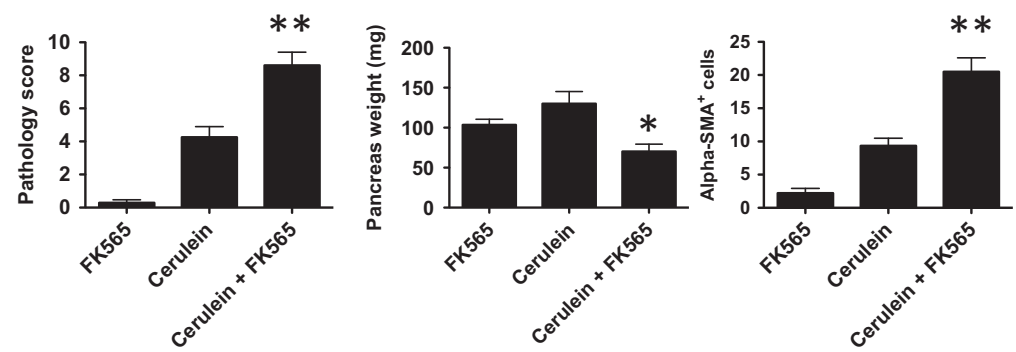

b H\&E CD3

CD11b

$\alpha-S M A$

Sirius red

Fibronectin
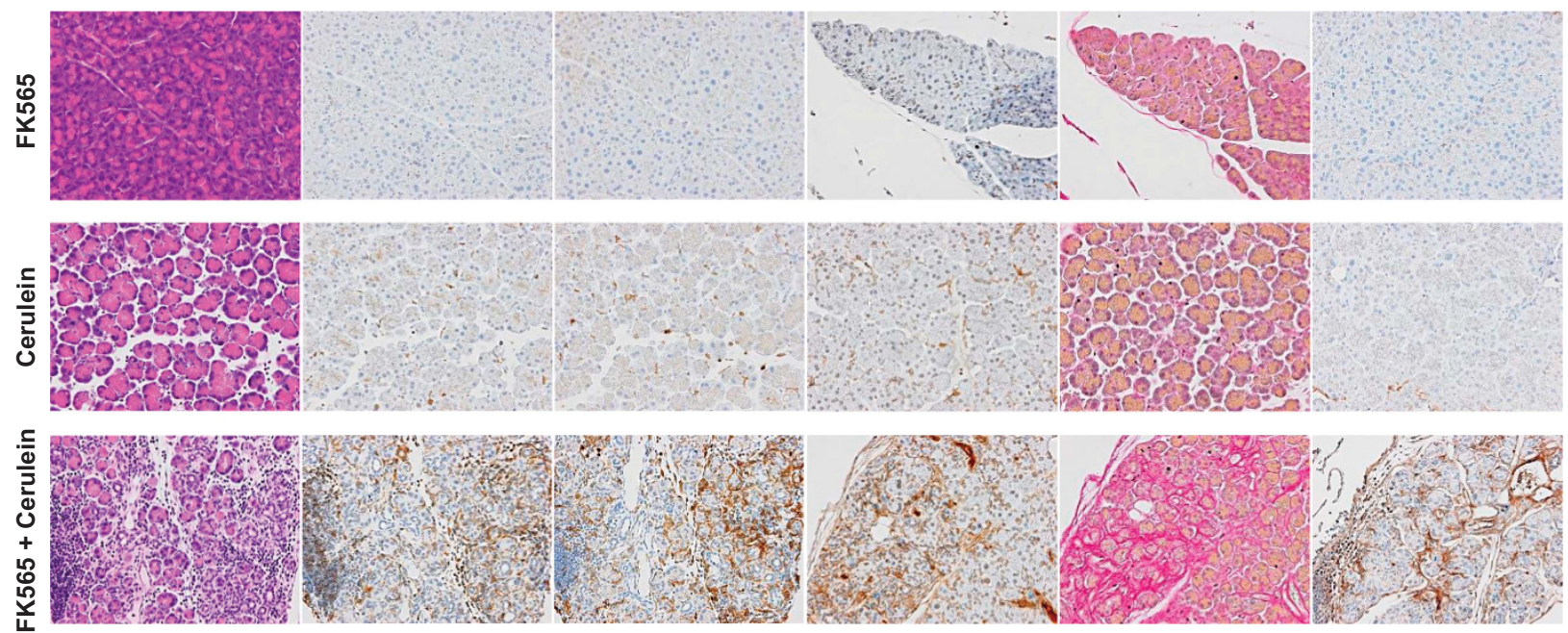

Figure 1 Induction of chronic pancreatitis in mice treated with NOD1 ligand and a low-dose cerulein. (a) Experimental protocol (Left): C57BL6 mice were administered FK565 (NOD1 ligand, $50 \mu \mathrm{g})$ alone $(n=7)$, a low-dose cerulein $\left(20 \mu \mathrm{g} \mathrm{kg}^{-1}\right)$ for a total of three times $(n=8)$, or FK565 followed by a lowdose cerulein $\left(20 \mathrm{~g} \mathrm{~kg} \mathrm{k}^{-1}\right)$ for a total of three times $(n=10)$. Mice received each regimen twice a week for a total of 14 times and then sera and pancreatic tissues were obtained. Serum levels of amylase, pathological scores of the pancreas, pancreatic weight, and the numbers of pancreatic $\alpha$-SMA ${ }^{+}$cells per high power fields obtained from mice $3 \mathrm{~h}$ after the last injection of cerulein. Results are expressed as mean \pm s.e.m. and are a pool of two independent experiments. ${ }^{\star} P<0.05,{ }^{\star *} P<0.01$ as compared with cerulein alone. (b) Representative picture of the pancreas tissue stained with hematoxylin and eosin (H\&E) staining, anti-CD3 Ab, anti-CD11b Ab, anti-SMA Ab, Sirius red, and anti-fibronectin Ab. Magnification $\times 400$. Ab, antibody; NOD1, nucleotidebinding oligomerization domain 1.

presence of $\mathrm{CD}^{+}$cells ( $\mathrm{T}$ cells) and $\mathrm{CD} 11 \mathrm{~b}^{+}$cells (myeloid cells such as macrophages), which showed very mild increases of these cells in mice administered cerulein alone but massive increases of these cells in mice administered the FK565-cerulein $\mathrm{CP}$ regimen. Moreover, as shown in Supplementary Figure S1b, flow-cytometric analysis revealed that total number of innate immune cells such as $\mathrm{CD}_{11} \mathrm{~b}^{+}$myeloid cells, $\mathrm{F} 4-80^{+}$macrophages, and $\mathrm{CD} 11 \mathrm{c}^{+}$dendritic cells was increased in the pancreas of mice treated with the FK565-cerulein CP regimen as compared with those treated with cerulein alone. In contrast, mice in both groups exhibited similar total numbers of pancreatic Gr- ${ }^{+}$granulocytes. Finally, in studies to evaluate the presence of pancreatic fibrosis, tissue was stained to detect pancreatic stellate cells (PSCs) expressing $\alpha$-smooth muscle actin (SMA), i.e., cells previously shown to be associated with pancreatic tissue fibrosis. ${ }^{20}$ Whereas tissue from mice administered cerulein alone exhibited only a barely detectable increase in $\alpha$-SMA ${ }^{+}$ cells, mice administered the FK565-cerulein CP regimen exhibited a large increase in $\alpha$-SMA ${ }^{+}$cells (Figure 1a,b). These findings were accompanied by robust staining of tissue from mice administered the FK565-cerulein CP regimen (but not administered cerulein alone) to detect the presence of collagen (with Sirius Red) and fibronectin. Taken together, these data offer strong evidence that the administration of the FK565-cerulein CP regimen induces chronic pancreatitis characterized by disruption of acinar architecture, massive infiltration of immune cells, and fibrosis.

\section{Chronic pancreatitis induced by the administration of the FK565-cerulein CP regimen requires NOD1 signaling and downstream induction of type I interferon}

In previous studies, we showed that development of acute pancreatitis induced by a single injection of NOD1 ligand and low-dose cerulein depends on the activation of the NOD1 signaling pathway accompanied by its downstream induction of type I interferon (IFN) and activation of the type I IFN signaling pathway. ${ }^{15}$ We therefore determined whether chronic pancreatitis in the model described above develops in mice deficient in these pathways due to NOD1 or type I IFN receptor (IFNAR) gene deletions. As shown in Figure 2a, and Supplementary Figures S2a and S2b, serum levels of 
a

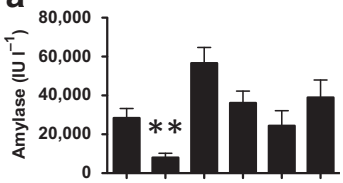

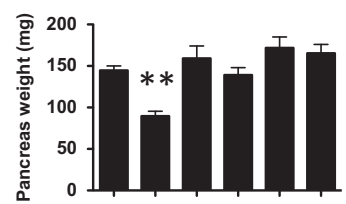

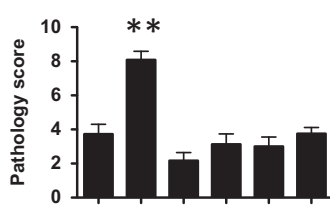

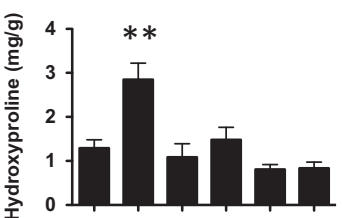

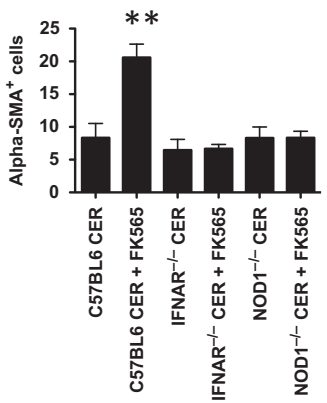

b

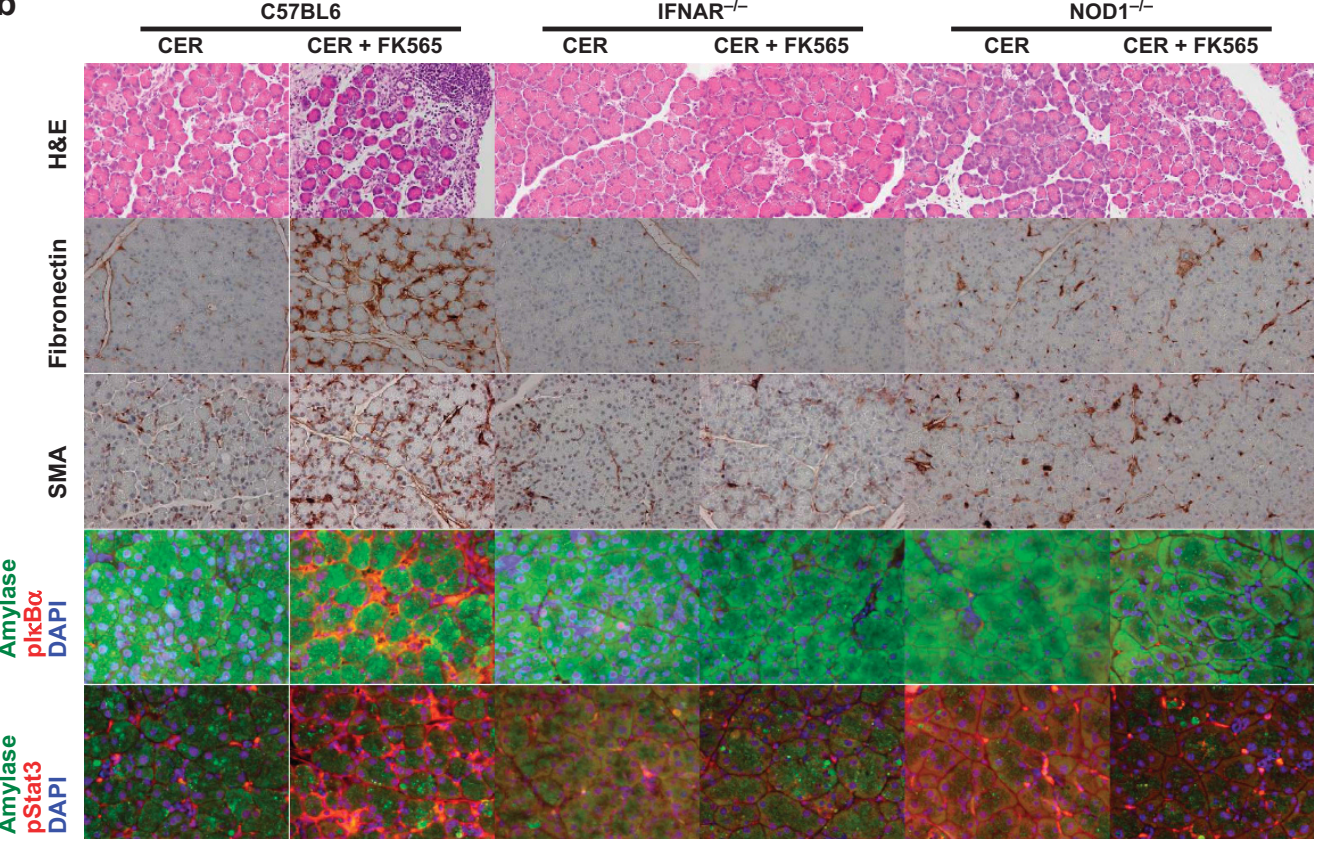

C

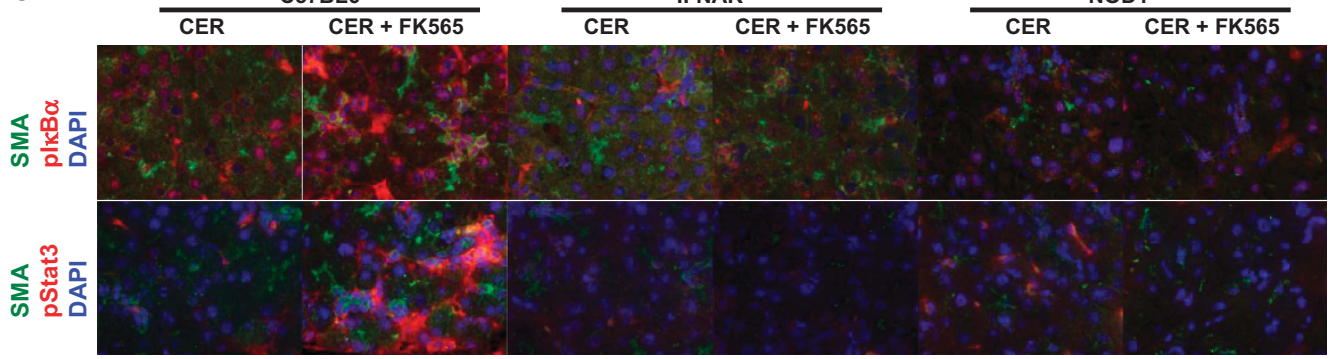

Figure 2 Induction of chronic pancreatitis in mice treated with NOD1 ligand and a low-dose cerulein requires intact NOD1 and type I IFN receptor signaling pathways. (a) C57BL6 mice, IFNAR-deficient mice (IFNAR ${ }^{-1-}$ ), or NOD1-deficient mice (NOD1 ${ }^{-1-}$ ) were administered a low-dose cerulein for a total three of times, or FK565 followed by a low-dose cerulein for a total of three times as described in Figure 1. Mice received each regimen twice a week for a total of 14 times and then sera and pancreatic tissues were obtained. Total numbers of mice in each group were as follows; C57BL6 cerulein (CER): $n=11$, C57BL6 CER + FK565: $n=11$, IFNAR ${ }^{-1}$ CER: $n=6$, IFNAR ${ }^{-1}$ CER + FK565: $n=8$, NOD1 ${ }^{-1-}$ CER: $^{-} n=10$, NOD1 $^{-1}$

CER + FK565: $n=8$. Serum levels of amylase, pancreatic weight, pathological scores of the pancreas, pancreatic levels of hydroxyproline, and the numbers of pancreatic $\alpha-\mathrm{SMA}^{+}$cells per high-power fields obtained from mice $3 \mathrm{~h}$ after the last injection of cerulein. Results are expressed as mean \pm s.e.m. and are a pool of two independent experiments. ${ }^{*} P<0.01$ as compared with cerulein alone in each group. (b) Representative picture of the pancreas tissue stained with H\&E, anti-fibronectin and anti-SMA, magnification $\times 400$ (top three lines). Representative picture of dual

immunofluorescence of the pancreas tissue stained with anti-amylase Ab (green color) or anti-pl $\kappa \mathrm{B} \alpha \mathrm{Ab}$ (red color) or anti-pStat3 Ab (red color). Nuclei were stained with DAPI, magnification $\times 800$ (bottom two lines). (c) Representative picture of dual immunofluorescence of the pancreas tissue stained with anti-SMA Ab (green color) or anti-plкB $\alpha$ Ab (red color) or anti-pStat3 Ab (red color). Nuclei were stained with DAPI, magnification $\times 1200$. H\&E; hematoxylin and eosin; IFN, iterferon.

amylase and pancreas weight were again significantly reduced and pathology scores were again increased in C57BL/6 mice administered the FK565-cerulein CP regimen as compared with mice administered repeated cerulein alone. However, these differences were abolished in mice with NOD1 or IFNAR deficiency since in this case the deficient mice subjected to administration of the FK565-cerulein CP regimen did not manifest more pancreatitis than the deficient mice subjected to cerulein treatment alone. This effect of NOD1 or IFNAR deficiency on induction of chronic pancreatitis was verified with tissue staining studies that showed that pancreatic tissue from wild-type mice and deficient mice administered cerulein alone were indistinguishable upon $\mathrm{H} \& \mathrm{E}$ staining as well as upon tissue staining to detect fibronectin or $\alpha$-SMA: in addition, as shown in Figure 2a,b, the FK565-cerulein $\mathrm{CP}$ regimen induced marked expression of pancreatic fibronectin and $\alpha$-SMA in wild-type mice, but not in NOD1 or IFNAR-deficient mice.

In further and separate studies to quantify the above findings, pancreatic fibrosis was assessed by a hydroxyproline assay and the number of $\alpha$-SMA ${ }^{+}$cells/high-powered field was determined. As shown in Figure 2a, these studies revealed that pancreatic levels of hydroxyproline were much higher in wild-type mice treated with FK565-cerulein $\mathrm{CP}$ regimen as 
compared with those treated with cerulein alone. In addition, no increases were seen in the pancreatic levels of hydroxyproline or in the number of $\alpha$-SMA ${ }^{+}$PSCs in NOD1- or IFNAR-deficient mice treated with cerulein alone or with the FK565-cerulein CP regimen as compared with wild-type mice treated with cerulein alone. Taken together, these data strongly suggest that the development of chronic pancreatitis induced by the administration of the FK565-cerulein CP regimen requires intact NOD1 and type I IFN signaling pathways.

As also shown previously, development of acute pancreatitis induced by a single injection of NOD1 ligand and low-dose cerulein required activation of NF- $\kappa \mathrm{B}$ and signal transducer and activator of transcription 3 (Stat3) in acinar cells, each dependent on complementary NOD1 and cerulein signaling. To determine whether such activation of NF- $\kappa \mathrm{B}$ and Stat 3 also occurs in the chronic pancreatitis model developed here we analyzed the expression of phospho-IкB $\alpha \quad(\mathrm{pI \kappa B} \alpha)$ and phospho-Stat3 (pStat3) in the pancreas of C57BL/6 mice administered the FK565-cerulein CP regimen. As shown in Figure $\mathbf{2 b}$, expression of $\mathrm{pI \kappa B} \alpha$ was seen in both amylasepositive acinar cells as well as in amylase-negative cells localized in the peri-acinar cell space. In addition, expression of pStat3 was also detected in amylase-positive pancreatic acinar cells and in amylase-negative cells localized in the peri-acinar space, although in this case, expression in amylase-positive acinar cells was lower than in amylase-negative cells. As expected, in a view of their relative lack of pancreatic inflammation in response to administration of FK565-cerulein CP regimen, NOD1-, or IFNAR-deficient mice exhibited little if any $\mathrm{pI} \kappa \mathrm{B} \alpha$ and $\mathrm{pStat} 3$ expression.

The observation that cells residing in the peri-acinar cell space express both $\mathrm{pI} \mathrm{B} \alpha \boldsymbol{\alpha}$ and $\mathrm{pStat} 3$ prompted us to investigate whether these cells were the PSCs mentioned above that are known to contribute to pancreatic inflammation and fibrosis. As shown in Figure 2c, the number of peri-acinar $\alpha$-SMA ${ }^{+}$ PSCs expressing both $\mathrm{pI \kappa B} \alpha$ and pStat 3 was in fact increased in the pancreas of C57BL/6 mice administered the FK565-cerulein $\mathrm{CP}$ regimen as compared with those treated with cerulein alone. In contrast, $\alpha$-SMA ${ }^{+}$PSCs expressing $\mathrm{pI} \kappa \mathrm{B} \alpha$ and pStat 3 were barely seen in the pancreas of NOD1- or IFNAR-deficient mice regardless of the mode of treatment. Thus, administration of the FK565-cerulein CP regimen caused NOD1-type I IFN-dependent activation of PSCs as well as acinar cells.

\section{Expression of proinflammatory mediators is enhanced in chronic pancreatitis induced by administration of the FK565-cerulein CP regimen}

As repeated injection of FK565 and cerulein induces activation of signaling pathways mediated by NF- $\kappa \mathrm{B}$, Stat 3 , and type I IFN in the pancreas of $\mathrm{C} 57 \mathrm{BL} / 6$ mice, we determined the expression of proinflammatory mediators related to these pathways in mice with chronic pancreatitis. As shown in Figure 3a, expression of IFN- $\beta$ was markedly enhanced in pancreatic lysates of $\mathrm{C} 57 \mathrm{BL} / 6$ mice when mice were administered the FK565-cerulein CP regimen, but not when admin- istered cerulein alone. In contrast, administration of the FK565-cerulein $\mathrm{CP}$ regimen did not augment the expression of IFN- $\beta$ in NOD1- or IFNAR-deficient mice. Consistent with these results, administration of FK565-cerulein CP regimen augmented expression of CXCL9 and CXCL10, chemokines whose production depends upon type I IFN signaling. ${ }^{21}$ In a similar vein, pancreatic expression of NF- $\kappa B-$ related proinflammatory mediators such as CCL2, TNF- $\alpha$, and IL- 6 was enhanced in C57BL/6 mice treated with the FK565-cerulein CP regimen. Again, such increases were not seen in NOD1- or IFNAR-deficient mice, in the latter case because type I IFN production is necessary for the influx of proinflammatory myeloid cells that produce NF- $\kappa B$-dependent factors. As shown in Figure 3b, these pancreatic cytokine and chemokine production profiles were consistent with the elevated serum levels of IFN- $\beta$, CXCL9, CCL2, and IL-6 exhibited by mice administered FK565-cerulein CP regimen but not cerulein alone.

As shown in Figure 3a, a similar picture was obtained with respect to cytokines usually produced in adaptive immune responses inasmuch as administration of the FK565-cerulein $\mathrm{CP}$ regimen markedly enhanced the pancreatic expression of IFN- $\gamma$ in C57BL/ 6 mice, but not in NOD1- or IFNAR-deficient mice. In addition, pancreatic expression of Th2 cytokines such as IL-4 and IL- 5 was also enhanced in C57BL/6 mice treated with the FK565-cerulein CP regimen as compared with those with the cerulein alone, although the degree of enhancement was much smaller for these Th2 cytokines than for Th1 cytokines.

The above evaluation of soluble mediators induced by the administration of the FK565-cerulein CP regimen also included analysis of mediators associated with pancreatic fibrosis. This included determination of pancreatic lysate and serum levels of TGF- $\beta 1$, IL-33, and IL-13, factors that have been previously identified as inducers of hepatic and intestinal fibrosis. ${ }^{22,23}$ Thus, as shown in Figure 3a, pancreatic lysates of C57BL/6 mice administered the FK565-cerulein CP regimen, but not mice administered cerulein alone, exhibited markedly enhanced expression of IL-33, IL-13, and TGF- $\beta 1$ and this enhanced expression was also evident in the level of serum IL-33. Again, such enhanced expression was not seen in the pancreatic lysates of NOD1- or IFNAR-deficient mice. Finally, as shown in Supplementary Figure S2c,d, IL-33 was highly expressed in C57BL/6 mice administered the FK565-cerulein CP regimen but not mice administered cerulein alone and such expression was not seen in mice deficient in IFNAR and NOD1. Taken together, these data indicate that administration of the FK565-cerulein CP regimen induces NOD1- and type I IFN-dependent expression of proinflammatory and profibrogenic mediators in the pancreas of mice.

\section{Chronic pancreatitis requires NOD1 expression in non-hematopoietic cells}

Having defined the cytokine and chemokine profiles accompanying chronic pancreatitis induced by the administration of 

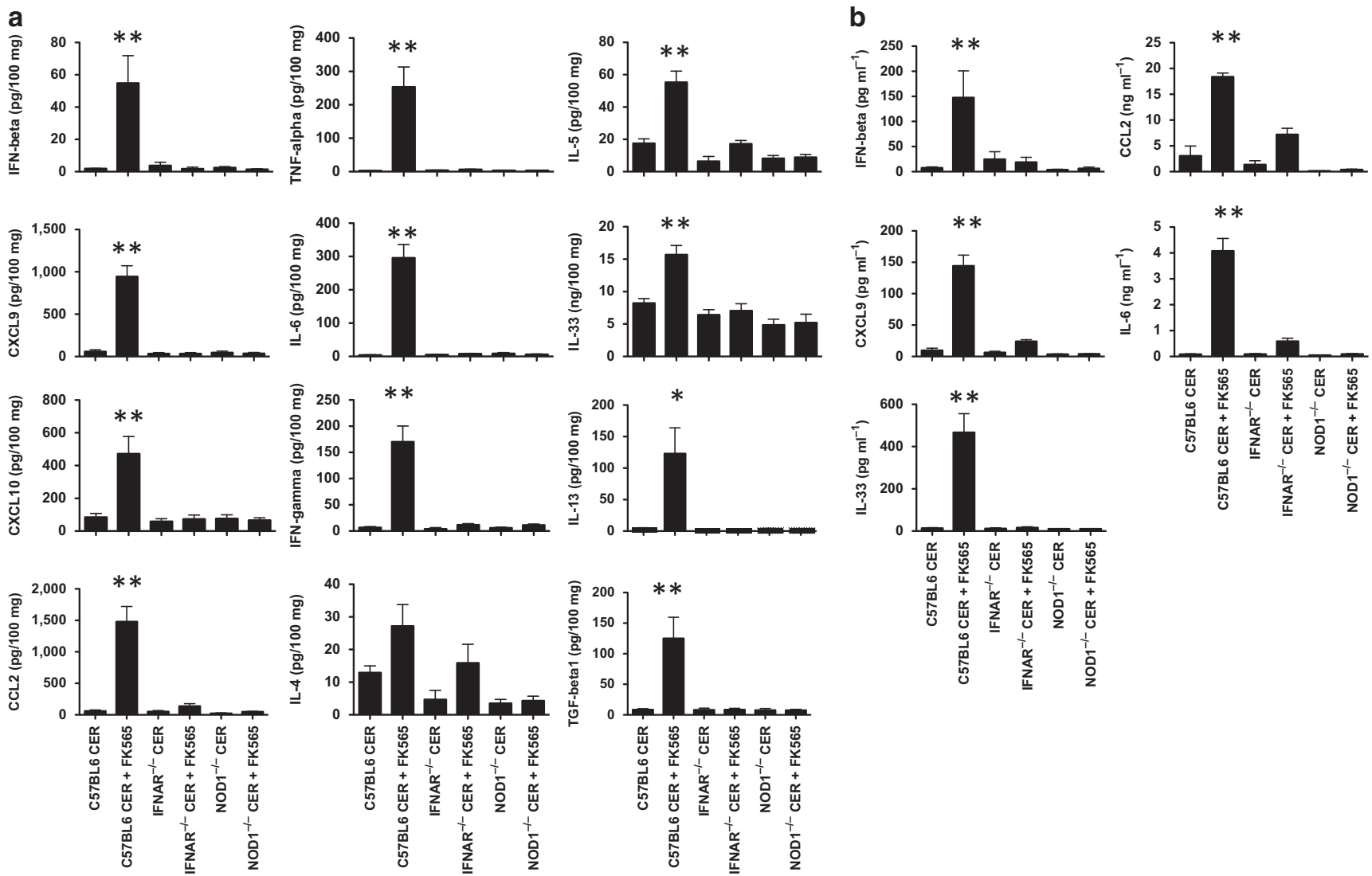

Figure 3 Profiles of cytokines and chemokines expression in mice treated with a low-dose cerulein and NOD1 ligand. C57BL6 mice, IFNAR-deficient $\left(\right.$ IFNAR $^{-1-}$ ), or NOD1-deficient (NOD1 ${ }^{-l-}$ ) mice were administered a low-dose cerulein (CER) for a total of three times or FK565 followed by a low-dose cerulein for a total of three times as described in Figure 2. Mice received each regimen twice a week for a total of 14 times and then sera and pancreatic lystes were prepared. Concentrations of cytokines and chemokines in pancreatic lysates (a) and serum (b) were determined by ELISA. Levels of cytokines and chemokines in the pancreas are shown as values per $100 \mathrm{mg}$ pancreatic tissue. Results are expressed as mean \pm s.e.m. and are a pool of two independent experiments. ${ }^{*} P<0.05,{ }^{\star *} P<0.01$ as compared with cerulein alone. ELISA, enzyme-linked immunosorbent assay; IFN, interferon.

the FK565-cerulein CP regimen, we turned our attention in defining the cellular origin of NOD1 and type I IFN signaling shown above to be necessary for this inflammation. NOD1 is expressed in hematopoietic cells such as antigen presenting cells as well as in non-hematopoietic cells such as pancreatic acinar cells. ${ }^{13}$ To determine whether NOD1 was acting in hematopoietic cells and/or non-hematopoietic cells in the chronic pancreatitis model induced by administration of the FK565-cerulein CP regimen we conducted studies of pancreatitis induction in bone marrow (BM)-chimeric mice. The latter consisted of irradiated NOD1-intact green fluorescent protein (GFP)-transgenic mice and irradiated NOD1-deficient mice reconstituted with NOD1-deficient GFP transgene-negative BM cells and NOD1-intact GFP transgene-positive $\mathrm{BM}$ cells that were prepared according to our previous report. ${ }^{15}$

As shown in Figure 4a and Supplementary Figure S3a, serum levels of amylase was significantly lower and pathology score was significantly higher in NOD1-intact mice transplanted with NOD1-deficient BM cells, but not in NOD1-deficient mice transplanted with NOD1-intact BM cells upon administration of the FK565-cerulein $\mathrm{CP}$ regimen, as compared with, similarly reconstituted mice administered cerulein alone. In addition, as shown in Figure $\mathbf{4 b}$, serum levels of IFN- $\beta$, CXCL9, IL-33, and CCL2 were significantly higher in NOD1-intact mice transplanted with NOD1-deficient BM cells, but not NOD1-deficient mice transplanted with NOD1intact BM cells, upon administration of the FK565-cerulein CP regimen, as compared with similarly reconstituted mice administered cerulein alone. As shown in Figure 4a,c, these findings were consistent with tissue staining studies that showed that NOD1-intact mice transplanted with NOD1-deficient BM cells, but not NOD1-deficient mice transplanted with NOD1-intact BM cells displayed increased expression of fibronectin, $\alpha$-SMA, and IL-33 in pancreatic tissue following administration of the FK565-cerulein CP regimen vs. cerulein alone.

In further studies of $\mathrm{BM}$-chimeric mice we examined the cellular location of $\mathrm{pI \kappa B} \alpha$ and pStat3 in mice with chronic pancreatitis. To this end, we first established that amylase-expressing pancreas acinar cells were GFP-positive in irradiated NOD1-intact GFP-transgene-positive mice 
a

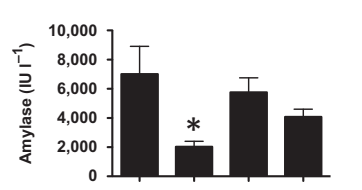

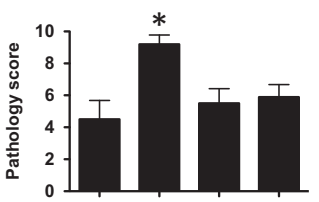

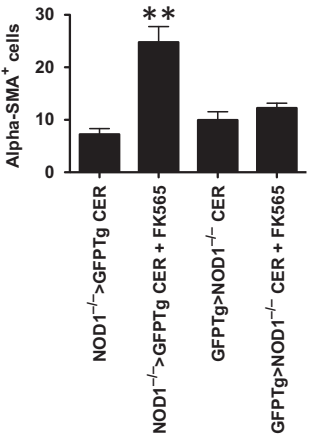

b
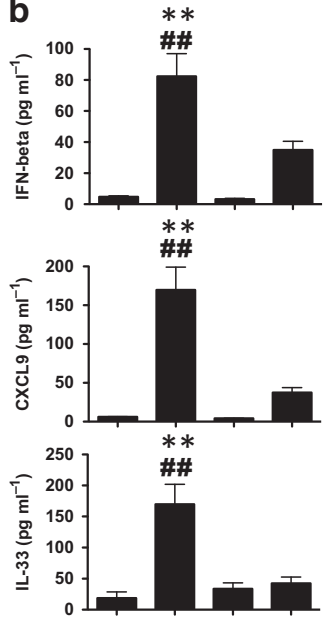

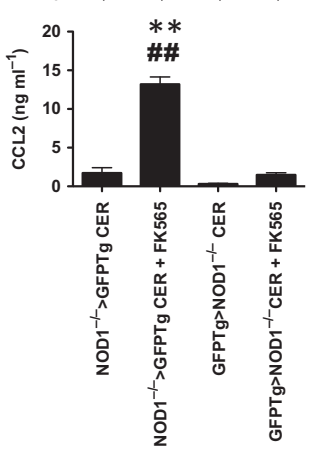

C

C

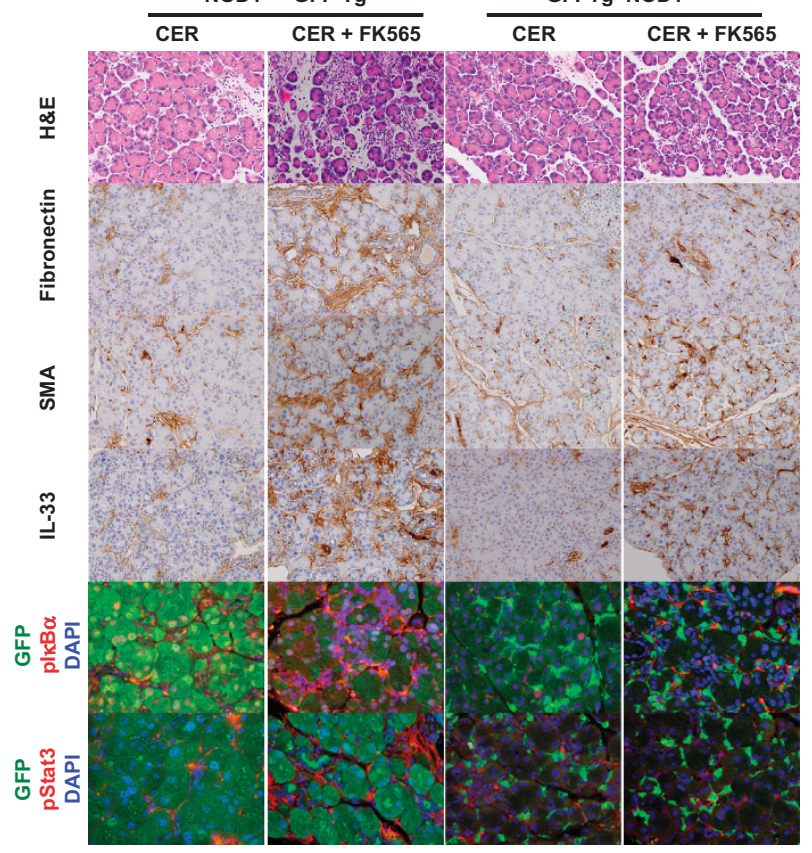

Figure 4 NOD1 expression in pancreatic acinar cells is necessary for the development of chronic pancreatitis induced by a low-dose cerulein and NOD1 ligand. Irradiated GFP-transgenic (GFP-Tg) NOD1-intact mice and NOD1-deficient mice were reconstituted with bone marrow (BM) cells from NOD1-deficient mice and GFP-Tg mice, respectively. These mice were referred to as NOD1 ${ }^{-1-}>$ GFP Tg and GFPTg $>$ NOD $1^{-1-}$, respectively. $\mathrm{NOD}^{-1-}>$ GFP Tg mice and GFPTg $>$ NOD1 ${ }^{-1-}$ mice were administered a low-dose cerulein for a total of three times, or FK565 followed by a lowdose cerulein for a total of three times as described in Figure 1. Mice received each regimen twice a week for a total of 11 times and then sera and pancreatic tissues were obtained. Total numbers of mice in each group were as follows; NOD1 ${ }^{-1-}>$ GFP Tg cerulein (CER): $n=6$, NOD1 ${ }^{-l-}>$ GFP Tg CER + FK565: $n=5$, GFPTg > NOD1 ${ }^{-1}$ CER: $n=5$, GFPTg $>$ NOD1 ${ }^{-1}-$ CER + FK565: $n=9$. (a) Serum levels of amylase, pathological scores of the pancreas, and the numbers of pancreatic $\alpha-\mathrm{SMA}^{+}$cells per high-power fields obtained from mice three hours after the last injection of cerulein. Results are expressed as mean \pm s.e.m. and are a pool of three independent experiments. ${ }^{\star} P<0.05,{ }^{\star \star} P<0.01$ as compared with cerulein alone. (b) Concentrations of cytokines and chemokines in the serum were determined by ELISA. Results are expressed as mean \pm s.e.m. and are a pool of three independent experiments. ${ }^{* *} P<0.01$ as compared with NOD1 ${ }^{-1-}>$ GFP Tg mice treated with cerulein alone, ${ }^{\# \#} P<0.01$ as compared with GFPTg $>$ NOD $1^{-1-}$ mice treated with cerulein and FK565. (c) Representative picture of the pancreas tissue stained with H\&E, anti-fibronectin Ab, antiSMA Ab or anti-IL-33 Ab magnification $\times 400$ (top four lines). Representative picture of dual immunofluorescence of the pancreas tissue stained with antiGFP Ab (green color) or anti-plkB $\alpha$ Ab (red color) or anti-pStat3 Ab (red color). Nuclei were stained with DAPI, magnification $\times 800$ (bottom two lines). GFP, green fluorescent protein; H\&E; hematoxylin and eosin; NOD1, nucleotide-binding oligomerization domain 1.

reconstituted with GFP-negative NOD1-deficient BM cells, whereas they were GFP-negative in irradiated NOD1-deficient GFP-transgene-negative mice reconstituted with GFP-positive NOD1-intact BM cells (data not shown). Thus, the status of GFP expression in acinar cells reflected the GFP status of the recipient. As shown in Figure 4c, expression of $\mathrm{pI \kappa B} \alpha$ was markedly enhanced not only in the GFP-positive acinar cells but also in the GFP-negative hematopoietic cells in the pancreas of irradiated NOD1-intact GFP-transgenepositive mice reconstituted with GFP-negative NOD1-deficient BM cells upon administration of the FK565-cerulein CP regimen. In addition, expression of pStat 3 was markedly enhanced in GFP-negative hematopoietic cells in the pancreas of irradiated NOD1-intact GFP-transgene-positive mice reconstituted with GFP-negative NOD1-deficient BM cells upon administration of the FK565-cerulein CP regimen as compared with reconstituted mice administered cerulein alone. In contrast, such expression of $\mathrm{pI \kappa B} \alpha$ and pStat3 was barely seen in the pancreas of irradiated NOD1-deficient GFP-transgene-negative mice reconstituted with GFP-positive NOD1-intact BM cells upon administration of the FK565-cerulein CP regimen. Collectively, the above data obtained from BM chimeric mice strongly suggest that NOD1 expression in non-hematopoietic cells, i.e., pancreatic acinar cells, is necessary for the development of chronic pancreatitis.

\section{Chronic pancreatitis is associated with-type I IFN receptor expression in non-hematopoietic or hematopoietic cells}

We next conducted additional BM chimera studies to determine whether type I IFN receptor (IFNAR) was acting in hematopoietic cells and/or non-hematopoietic cells in the 
development of the chronic pancreatitis developing in mice administered the FK565-cerulein CP regimen. BM-chimeric mice consisting of irradiated IFNAR-intact GFP-transgenic positive mice and irradiated IFNAR-deficient mice, reconstituted with IFNAR-deficient GFP-transgene-negative BM cells and IFNAR-intact GFP-transgene-positive BM cells, respectively, were prepared. As shown in Supplementary Figures S3b and S4a, the administration of the FK565-cerulein $\mathrm{CP}$ regimen induced equivalent levels of chronic pancreatitis as judged by pathology scores in both irradiated IFNAR-intact GFP-transgene-positive mice and irradiated IFNAR-deficient mice reconstituted with IFNAR-deficient GFP transgenenegative BM cells and IFNAR-intact GFP transgene-positive $\mathrm{BM}$ cells, respectively, as compared with both types of BM chimeric mice administered cerulein alone. As shown in Supplementary Figure S4b, consistent with the pathology scores, serum levels of IFN- $\beta$, CXCL9, IL-33, and CCL2 were significantly higher in both types of $\mathrm{BM}$ chimeric mice administered the FK565-cerulein CP regimen as compared with those treated with cerulein alone. Furthermore, as shown in Supplementary Figures S4a,c, administration of the FK565cerulein $\mathrm{CP}$ regimen but not cerulein alone induced pancreatic expression of fibronectin, $\alpha$-SMA, and IL-33 in both types of BM chimeric mice. These data suggest that type I IFNR expression in non-hematopoietic or hematopoietic cells is sufficient for the development of chronic pancreatic inflammation induced by administration of the FK565-cerulein CP regimen.

\section{IL-33 production by acinar cells is regulated by pancreatic myeloid cells}

As shown above (Figure 3) as well as in a previous study of experimental pancreatitis induced by bile duct blockade, ${ }^{24,25}$ pancreatitis is associated with an increased expression of IL-33. These findings, plus the fact that IL-33 is released by cells undergoing necrosis and acinar cell necrosis is a central feature of chronic pancreatitis, suggested to us that this cytokine has an important if not key role in the pathogenesis of chronic pancreatitis induced by the administration of the FK565-cerulein CP regimen.

In initial studies addressing the origin of IL-33 in this model, we considered the possibility that IL-33 was being produced by acinar cells or PSCs, the cell mentioned above that has been implicated in the development of pancreatic fibrosis and shown previously to express nuclear IL-33. ${ }^{26}$ Indeed, as shown in Supplementary Figure S2c, IL-33 expression was observed in the peri-acinar cell space of the pancreas where PSCs are found in C57BL/6 mice administered the FK565-cerulein CP regimen. However, as also shown in Supplementary Figure S2d, dual immunofluorescence studies revealed that most $\alpha$-SMA ${ }^{+}$PSCs were negative for IL-33 staining. This suggested that PSCs are not the main cellular source of this cytokine and that IL-33 in the peri-acinar space originates from necrotic acinar cells.

To further investigate pancreatic acinar cell production of IL-33, we determined the effect of cerulein and/or FK565 on such production in cultures of pancreatic acinar cells obtained from wild-type mice or acinar cells obtained from mice that had been subjected to the FK565-cerulein CP regimen. As shown in Supplementary Figure S5a, stimulation of wild-type acinar cells with either cerulein or FK565 induced increased production of IL-33 and the two stimuli acting together induced enhanced IL-33 production. Furthermore, such production was reduced in cells from either IFNAR- or NOD1-deficient mice. These data suggest that type I IFN produced by NOD1 ligand and/or cerulein-stimulated wildtype acinar cells leads to a modest level of IL-33 production by acinar cells. As shown in Supplementary Figure S5b, acinar cells obtained from the pancreas of a mouse that had been subjected in vivo to the FK565-cerulein $\mathrm{CP}$ regimen produced IL-33 at a level similar to those of naive wild-type acinar cells cultured with NOD1 ligand and cerulein; however, when cocultured with $\mathrm{CD} 1 \mathrm{~b}^{+}$myeloid cells they produced strikingly increased amounts of IL-33, which returned to baseline in the presence of anti-TNF- $\alpha$ Ab or anti-IFNAR Ab. This suggests that type I IFN is acting on myeloid cells to produce TNF- $\alpha$ and the latter is inducing acinar cells to produce large amounts of IL33. Taken together, these data suggest that pancreatic acinar cells are the main producers of IL-33 in this model of chronic pancreatitis and that pancreatic myeloid cells augment such IL33 production through a TNF- $\alpha$-mediated signaling pathway dependent on type I IFN signaling.

\section{IL-33 has a critical role in the chronic pancreatic inflammation induced by the administration of the FK565-cerulein CP regimen}

Previous studies have shown that IL-33 acts through ST2 to induce a wide variety of proinflammatory cytokines via a MyD88 signaling pathways and thus promotes the development of both inflammation and fibrosis. ${ }^{23,27,28} \mathrm{We}$ therefore investigated the role of IL-33 in the pathogenesis of chronic pancreatitis, induced by the administration of the FK565-cerulein CP regimen, with studies in which IL-33 function is blocked by inhibition of ST2 signaling. To this end, we determined the extent of pancreatitis in C57BL/6 mice administered the FK565-cerulein CP regimen or cerulein alone and treated with control $\mathrm{Ab}$ or neutralizing anti-ST2 $\mathrm{Ab} .^{29}$

As shown in Figure 5a and Supplementary Figure S6a, serum levels of amylase and pancreatic weight were significantly higher in mice administered the FK565-cerulein CP regimen and treated with anti-ST2 Ab as compared with mice treated with control $\mathrm{Ab}$ and the severity of pancreatitis as evaluated by pathology score in these mice was significantly reduced. In addition, as shown in Figure $\mathbf{5 a}, \mathbf{b}$, mice administered the FK565-cerulein CP regimen and treated with anti-ST2 Ab exhibited a pancreatitis profile similar to that in mice administered cerulein alone and control $\mathrm{Ab}$ as judged by serum levels of amylase and pathology score. Thus, anti-ST2 $\mathrm{Ab}$ treatment of mice administered the FK565-cerulein CP regimen exhibited the less severe pancreatitis and reduced pancreatic fibrosis characteristic of mice administered cerulein alone and control antibody.

As shown in Figure 5c, consistent with this amelioration in the level of inflammation and fibrosis, pancreatic extracts of 


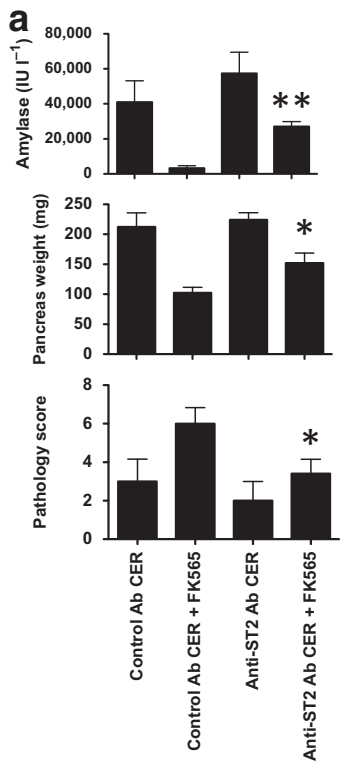

b

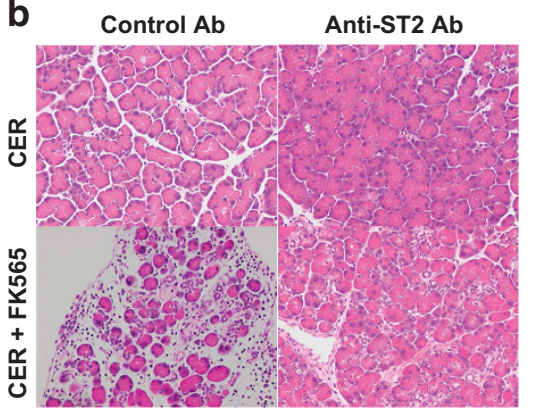

C
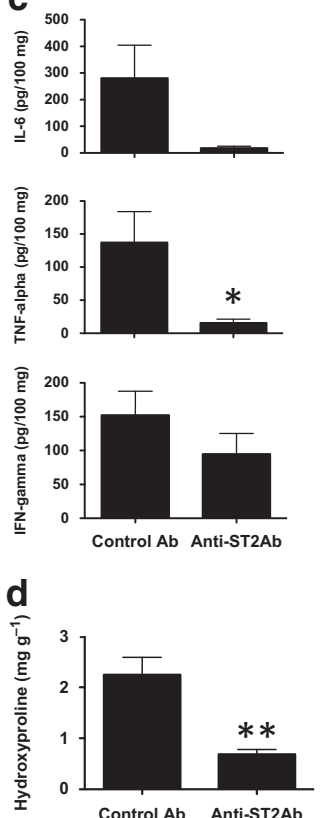

Control Ab Anti-ST2Ab
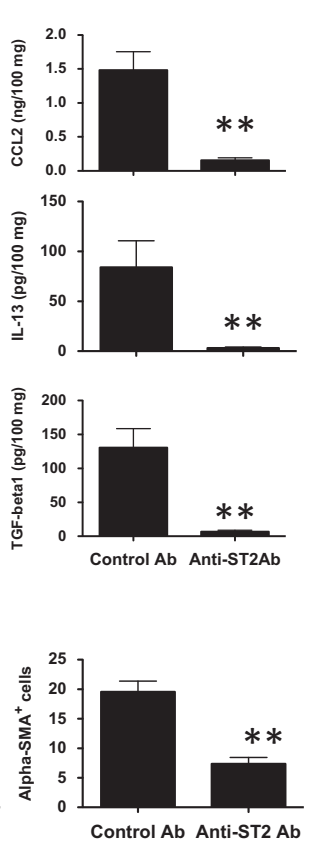

e

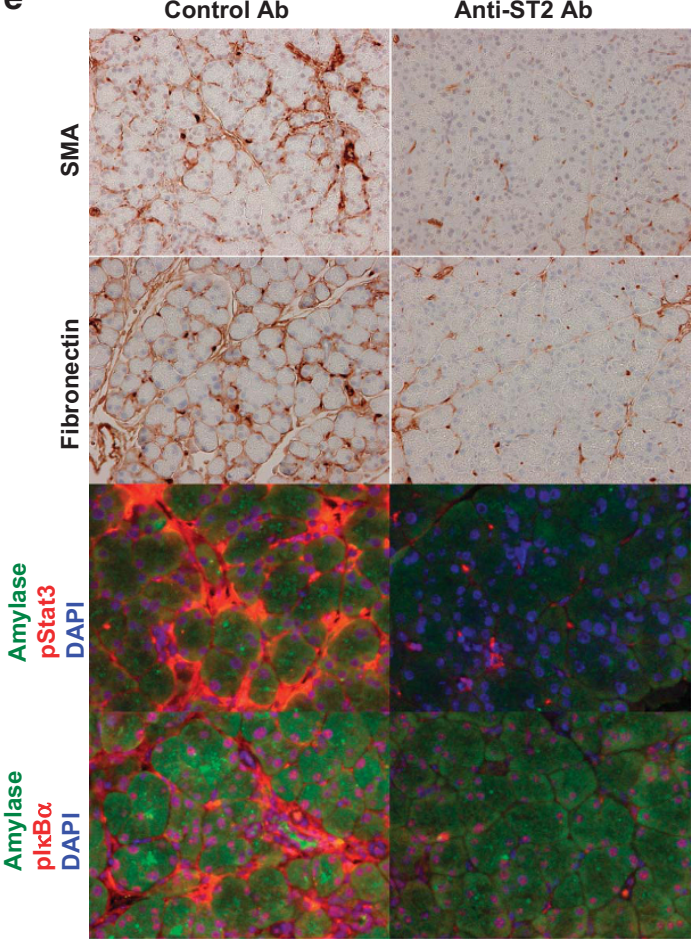

Figure 5 The interaction between IL-33 and ST2 is necessary for the development of chronic pancreatitis induced by a low-dose cerulein and NOD1 ligand. C57BL6 mice were administered a low-dose cerulein (CER) for a total of three times, or FK565 followed by a low-dose cerulein for a total of three times as described in Figure 1. Mice were also treated with intraperitoneal injection of control Ab (100 $\mu$ g, $n=3$; cerulein alone group, $n=5$; cerulein and FK565 group) or anti-ST2 antibody $(100 \mu \mathrm{g}, n=3$; cerulien alone group, $n=5$; cerulein and FK565 group) before the initiation of each treatment regimen. Mice received each regimen twice a week for a total of 14 times after which sera and pancreatic tissues were obtained. (a) Serum levels of amylase, pancreatic weight, and pathological scores of the pancreas obtained from mice three hours after the last injection of cerulein. Results are expressed as mean \pm s.e.m. ${ }^{*} P<0.05,{ }^{* *} P<0.01$ as compared with mice treated with control Ab, cerulein, and FK565. (b) Representative picture of the pancreas tissue stained with $\mathrm{H} \& \mathrm{E}$, magnification $\times 400$. (c) Concentrations of cytokines and chemokines in pancreatic lysates from mice treated with FK565-cerulein chronic pancreatitis regimen and control Ab or anti-ST2 Ab were determined by ELISA. Levels of cytokines and chemokines in the pancreas are shown as values per $100 \mathrm{mg}$ pancreatic tissue. Results are expressed as mean $\pm \mathrm{s}$.e.m. ${ }^{\star} P<0.05$, ${ }^{\star \star} P<0.01$ as compared with control Ab. (d) Pancreatic levels of hydroxyproline and the numbers of $\alpha$-SMA ${ }^{+}$cells in mice treated with FK565-cerulein chronic pancreatitis regimen and control Ab or anti-ST2 Ab. ${ }^{* *} P<0.01$ as compared with control Ab. (e) Representative picture of the pancreas tissue stained with anti-SMA Ab and anti-fibronectin $\mathrm{Ab}$, magnification $\times 400$. Representative picture of dual immunofluorescence of the pancreas tissue stained with anti-amylase Ab (green color) or anti-pStat3 Ab (red color) or anti-pl $\mathrm{B} \alpha \mathrm{Ab}$ (red color). Mice received FK565-cerulein chronic pancreatitis regimen with control Ab or anti-ST2 Ab. Nuclei were stained with DAPI, magnification $\times 800$. Ab, antibody; H\&E, hematoxylin and eosin; IL, interleukin; NOD1, nucleotide-binding oligomerization domain 1.

mice administered the FK565-cerulein CP regimen and treated with anti-ST2 Ab contained markedly decreased amounts of both proinflammatory mediators such as IL-6, TNF- $\alpha$, and CCL2 and profibrogenic cytokines such as IL-13 and TGF- $\beta 1$. In contrast, the same pancreatic extracts exhibited no significant reduction in IFN- $\gamma$ content. Furthermore, as shown in Figure 5d,e, consistent with the reduction in profibrogenic cytokines, pancreatic extracts from mice administered the FK565-cerulein CP regimen and treated with anti-ST2 Ab contained reduced levels of hydroxyproline, and pancreatic tissue from these mice exhibited reduced numbers of $\alpha-\mathrm{SMA}^{+}$cells and reduced fibronectin and $\alpha$-SMA-staining (Figure 5d,e). Finally, anti-ST2 Ab treatment led to reduced pancreatic tissue staining for detection of pStat 3 and $\mathrm{pI} \kappa \mathrm{B} \alpha$. Collectively, these studies show that blockade of IL-33-ST2 signaling inhibits the profibrotic features of the chronic pancreatic inflammation induced by administration of the FK565-cerulein CP regimen. 

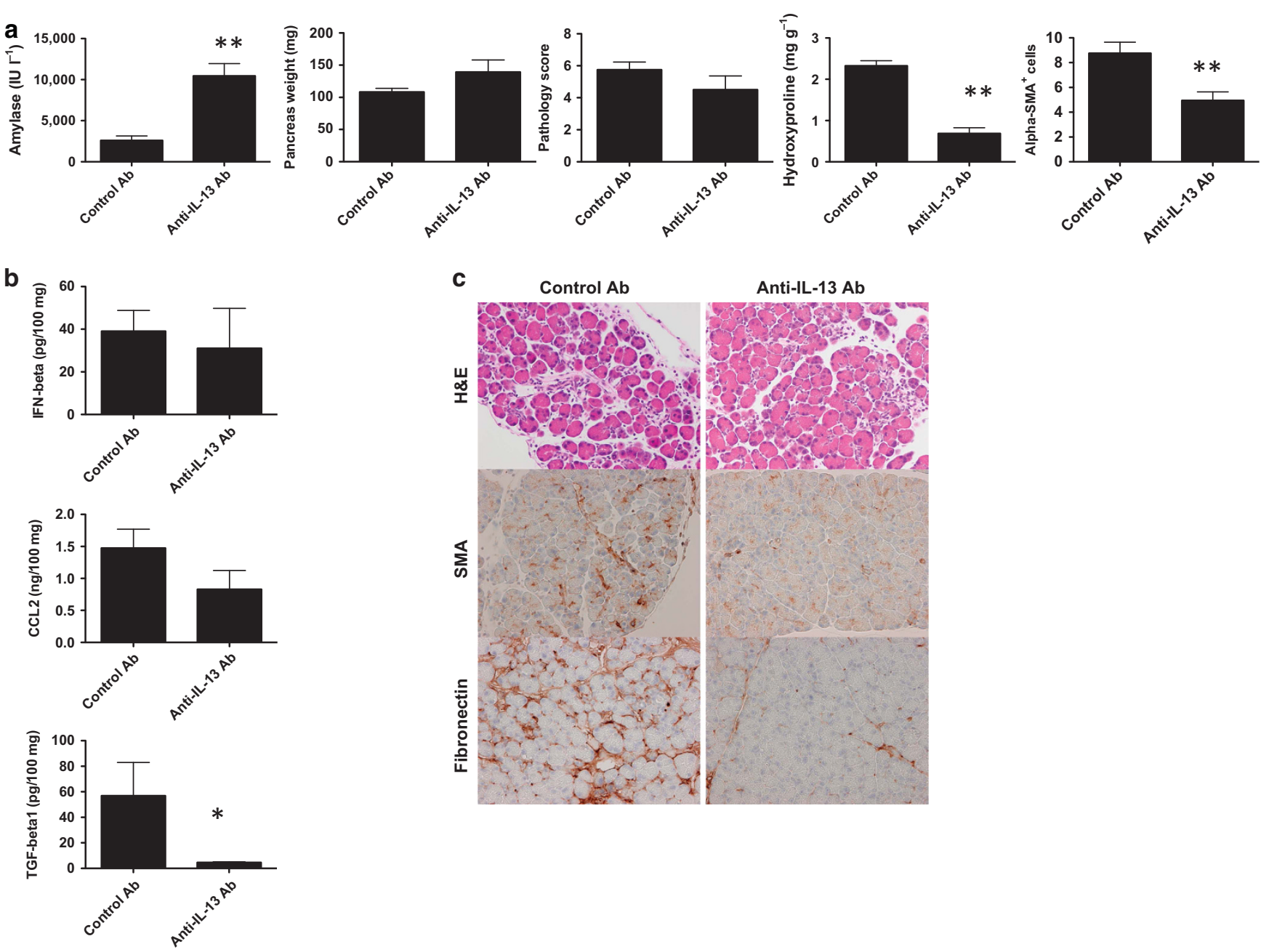

Figure 6 IL-13 is necessary for the development of pancreatic fibrosis induced by a low-dose cerulein and NOD1 ligand. C57BL6 mice were administered FK565 followed by a low-dose cerulein for a total of three times as described in Figure 1. Mice were also treated with intraperitoneal injection of control $\mathrm{Ab}(100 \mu \mathrm{g}, n=4)$ or anti-IL-13 antibody $(100 \mu \mathrm{g}, n=4)$ before the initiation of FK565-cerulein treatment regimen. Mice received each regimen twice a week for a total of 14 times and then sera and pancreatic tissues were obtained. (a) Serum levels of amylase, pancreatic weight, pathological scores of the pancreas, pancreatic levels of hydroxyproline, and the numbers of $\alpha$-SMA ${ }^{+}$cells obtained from mice $3 \mathrm{~h}$ after the last injection of cerulein Results are expressed as mean \pm s.e.m. ${ }^{* \star} P<0.01$ as compared with control Ab. (b) Concentrations of cytokines and chemokines in pancreatic lysates were determined by ELISA. Levels of cytokines and chemokines in the pancreas are shown as values per $100 \mathrm{mg}$ pancreatic tissue. Results are expressed as mean \pm s.e.m. ${ }^{*} P<0.05$ as compared with control Ab. (c) Representative picture of the pancreas tissue stained with H\&E, anti-SMA Ab and anti-fibronectin Ab, magnification $\times 400$. Ab, antibody; ELISA, enzyme-linked immunosorbent assay; H\&E; hematoxylin and eosin; IL, interleukin; NOD1, nucleotide-binding oligomerization domain 1.

\section{IL-13-mediated signaling pathways mediate fibrosis in chronic pancreatitis induced by the administration of the FK565-cerulein CP regimen}

As shown above, IL-33 signaling via ST2 induces IL-13 production and tissue fibrosis as shown by increased tissue expression of hydroxyproline and $\alpha$-SMA. This finding raised the possibility that IL-13 is a major profibrotic factor in the chronic pancreatitis induced by administration of the FK565-cerulein CP regimen. To investigate this possibility, C57BL/6 mice administered the FK565-cerulein CP regimen were treated with neutralizing $\mathrm{Ab}$ against IL-13 or control $\mathrm{Ab}$ at the time of each FK565-cerulein administration. As shown in Figure $\mathbf{6 a}, \mathbf{b}$ and Supplementary Figure S6b, serum levels of amylase and pancreatic weight was higher in mice treated with anti-IL-13 $\mathrm{Ab}$ as compared with mice treated with control Ab, but anti-IL-I3 Ab treatment had little effect on pathology score. These changes were not associated with changes in pancreatic extract content of IFN- $\beta$ or CCL2 but were associated with dramatic reduction in content of TGF- $\beta 1$ and decreases in pancreatic expression of $\alpha$-SMA, hydroxyproline, and fibronectin (Figure 6a-c). Thus, whereas IL-33 induction of IL-13 does not influence the severity of inflammation in this chronic fibrosis model, it is likely to be a major pathway by which IL-33 causes pancreatic fibrosis and atrophy.

\section{IL-13 induced by IL-33 in chronic pancreatitis originates mainly from $T$ cells}

Recent studies have shown that IL-33 activates innate lymphoid cell type 2 (ILC2) to promote IL-13-mediated tissue 
a

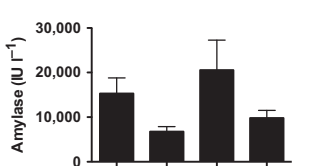

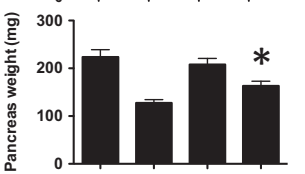

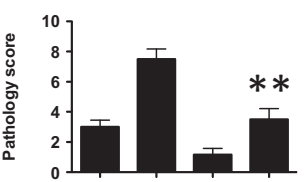

Tos 2.5

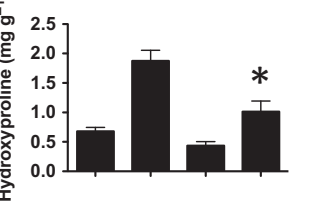

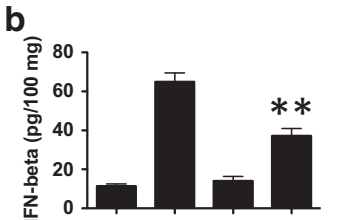

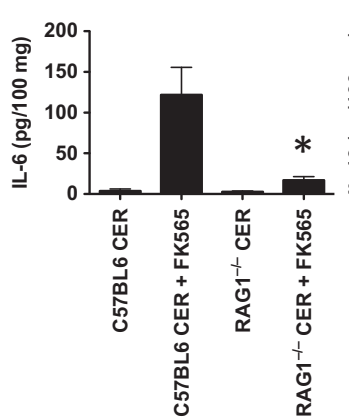

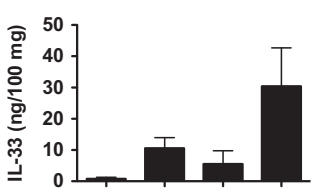

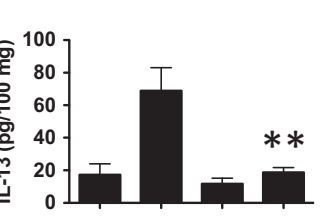

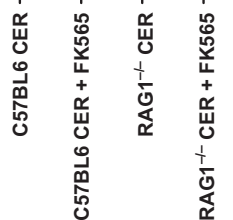

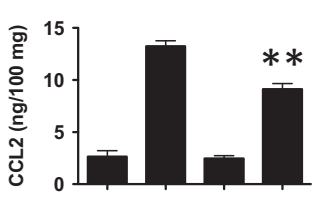

ิㅗㄹ 500
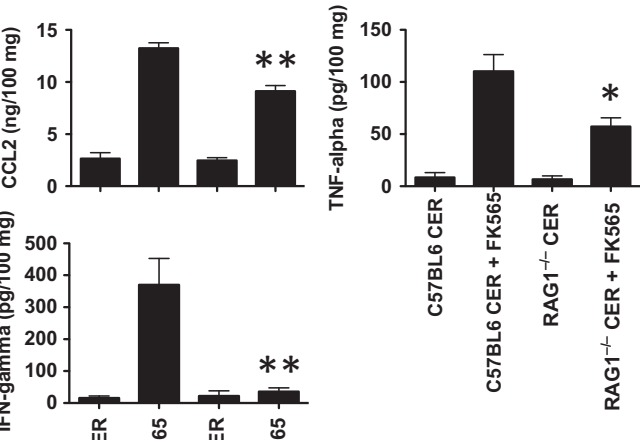

\section{C}

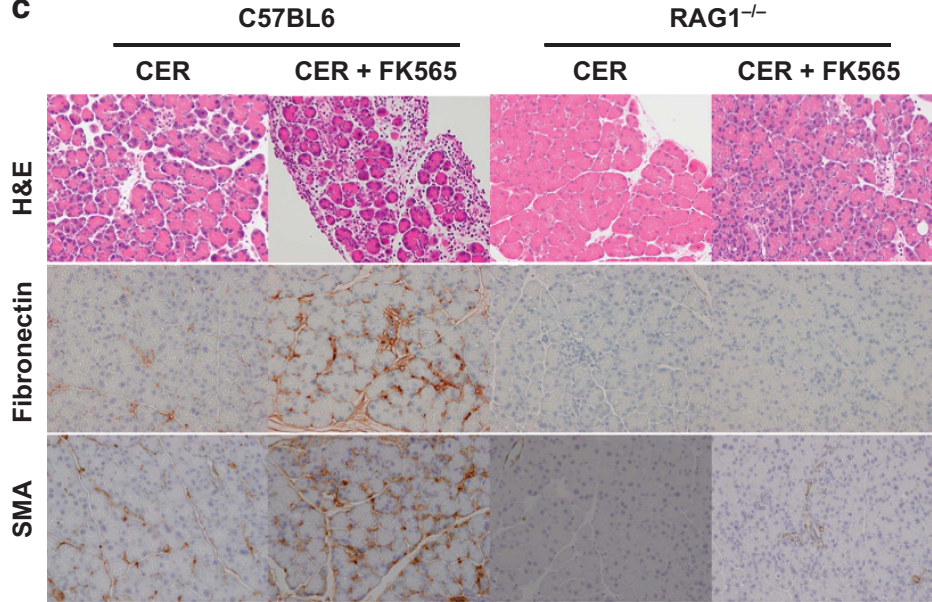

Figure 7 Development of chronic pancreatitis induced by NOD1 ligand and a low-dose cerulein requires adaptive immune responses (a) C57BL6 mice and RAG1-deficient mice (RAG1 ${ }^{-1-}$ ) were administered a low-dose cerulein for a total of three times, or FK565 followed by a low-dose cerulein for a total of three times as described in Figure 1. Mice received each regimen twice a week for a total of 14 times and then sera and pancreatic tissues were obtained. Total numbers of mice in each group were as follows; C57BL6 cerulein (CER): $n=9$, C57BL6 CER + FK565: $n=9$, RAG1 ${ }^{-1}$ - CER: $n=9$, RAG1 ${ }^{-1}$ - CER + FK565: $n=9$. Serum levels of amylase, pathological scores of the pancreas, pancreatic weight, pancreatic levels of hydroxyproline, and the numbers of $\alpha-\mathrm{SMA}^{+}$cells obtained from mice three hours after the last injection of cerulein. Results are expressed as mean \pm s.e.m. and are a pool of two independent experiments. ${ }^{*} P<0.05$, ${ }^{*} P<0.01$ as compared with C57BL6 mice treated with FK565 and cerulein. (b) Concentrations of cytokines and chemokines in pancreatic lysates were determined by ELISA. Levels of cytokines and chemokines in the pancreas are shown as values per $100 \mathrm{mg}$ pancreatic tissue. Results are expressed as mean \pm s.e.m. ${ }^{* *} P<0.01,{ }^{*} P<0.05$ as compared with C57BL6 mice treated with cerulein and FK565. (c) Representative picture of the pancreas tissue stained with H\&E, anti-fibronectin and anti-SMA, magnification $\times 400$. ELISA, enzyme-linked immunosorbent assay; H\&E; hematoxylin and eosin; NOD1, nucleotide-binding oligomerization domain 1.

fibrosis. ${ }^{23,27,30}$ We therefore conducted studies to determine if ILC2 and/or conventional T cells were the origin of IL-13 in the chronic pancreatitis induced by administration of the FK565-cerulein CP regimen. In initial studies addressing this question, we determined the type of cells producing IL-13 in the pancreas by dual immunofluorescence analysis and found that most of IL-13-producing cells were $\mathrm{CD}^{+}{ }^{+} \mathrm{T}$ cells (data not shown). Second, we isolated pancreatic mononuclear cells from C57BL/6 mice treated with the FK565-cerulein CP regimen and then pancreatic mononuclear cells were stimulated with anti-CD3 $\mathrm{Ab}$ and $\mathrm{CD} 28 \mathrm{Ab}$ to measure the production of IL-13. As shown in Supplementary Figure S7, CD $4^{+}$pancreatic mononuclear cells, but not $\mathrm{CD}^{+}$cells-depleted pancreatic mononuclear cells produced a large amount of IL-13.
Finally, in more definitive studies, we compared the chronic pancreatitis induced by administration of the FK565-cerulein $\mathrm{CP}$ regimen or repeated administration of cerulein alone in C57BL/6 mice to that induced in RAG-1-deficient mice lacking $\mathrm{T}$ cells. As shown in Figure 7 and Supplementary Figure S6c, differences in serum amylase levels between mice administered cerulein alone and those administered the FK565-cerulein CP regimen were generally equivalent in RAG1-deficient and wild-type mice; nevertheless, the RAG1-dificient mice exhibited a significant reduction in pathology score and increase in pancreatic weight as compared with the wild-type mice. In accompanying studies it was found that production of proinflammatory mediators produced in innate immune responses such as IFN- $\beta$, CCL2, TNF- $\alpha$, and IL- 6 was 
significantly reduced in the RAG1-deficient mice administered the FK565-cerulein CP regimen as compared with wild-type mice (Figure $7 \mathbf{b}$ ). Strikingly, production of proinflammatory mediators produced mainly in adaptive immune responses, IFN- $\gamma$ and IL-13, were even more decreased in the pancreas of RAG1-deficient mice administered the FK565-cerulein CP regimen. Thus, T-cell-mediated adaptive immune cytokines enhance innate immune-related proinflammatory cytokine responses to cause inflammation in the FK565-cerulein CP model. Finally, RAG-1-deficient mice manifested reduced expression of hydroxyproline, fibronectin, and $\alpha$-SMA upon administration of the FK565-cerulein CP regimen (Figure $7 \mathbf{a}, \mathbf{c}$ ). Thus, these data are compatible with the view that conventional $\mathrm{T}$ cells are mainly responsible for the development of both the persistent inflammation and the fibrosis in this chronic pancreatitis model. Nevertheless, it remains possible that ILC2 also contribute to the inflammation and further work exploring this possibility is necessary. Whether the adaptive IL-13 T-cell response was driven by pancreatic autoantigens or microbiome-related stimuli awaits further study.

\section{NOD1-deficient mice are resistant to the induction of chronic pancreatitis induced by high-dose cerulein}

The above studies establish a new model of chronic pancreatitis induced by the repeated administration of NOD1 ligand (FK565) and low-dose cerulein. However, since low-dose administration of cerulein was incapable of inducing pancreatitis on its own, it remained possible that NOD1 ligand administration was acting as an adjuvant that is merely enhancing the low-dose cerulein effect and does not have a unique pathogenic role. This possibility was rendered unlikely by our previous studies showing that high-dose cerulein administration is a poor inducer of acute pancreatitis in NOD1-deficient mice. ${ }^{15}$ Despite these findings, we conducted studies to determine whether high-dose cerulein administration can induce chronic pancreatitis in the absence of NOD1 signaling and thus under these circumstances where NOD1 signaling may be dispensable. Accordingly, C57BL/6 mice and NOD1-deficient mice were subjected to four hourly injection of high doses of cerulein $\left(100 \mu \mathrm{g} \mathrm{kg}^{-1}\right)$ twice a week for a total of 7 weeks. As shown in Supplementary Figure S8, NOD1-deficient mice were resistant to the induction of chronic pancreatitis by high-dose cerulein as assessed by chronic pancreatitis scores and induction of high pancreatic hydroxyproline levels. Perhaps more importantly, pancreatic expression of IL-33 was strikingly lower in NOD1-deficient mice as compared with wild-type mice. These data thus provide further support for the unique and indispensable role of the NOD1mediated innate immune response in the development of chronic pancreatitis.

\section{DISCUSSION}

In this manuscript we describe a new model of chronic pancreatitis induced by the repeated administration of NOD1 ligand (FK565) and low-dose cerulein. Importantly, both
NOD1 ligand and cerulein administration were essential to the induction of the chronic pancreatitis since NOD1 ligand alone did not induce any pancreatic inflammation and administration of cerulein alone led to a mild pancreatic inflammation that lacked the key feature of the chronic disease such as pancreatic atrophy and fibrosis. Given the pivotal role of NOD1 as a pattern recognition receptor responding to intestinal microflora, the findings in this study support the notion that NOD1-mediated innate immune responses occurring in acinar cells following their exposure to circulating bacteria are a necessary condition for the development of chronic pancreatitis initiated by a cause of excessive trypsinogen activation, such as cerulein administration.

Using this model, we were able to identify key proinflammatory mediators responsible for the innate and adaptive immune responses involved in the development of chronic pancreatitis. At first sight, the most obvious of these was NOD1 itself, as, as alluded to above, it was necessary to include NOD1 ligand in the pancreatitis-inducing regimen. However, it could be argued that the role of NOD1 in this model was to act as an enabler of the effect of low-dose cerulein administration as the latter was incapable of inducing pancreatitis on its own and NOD1 does not itself have a unique pathogenic role in the inflammation. This possibility, however, does not fit with the observation that NOD1 stimulation of acinar cells was necessary for the production of type I IFN and other factors shown to be involved in the inflammation (see further discussion below). Perhaps more importantly, this possibility was ruled out by the fact that even high-dose cerulein administration was not able to induce full-blown chronic pancreatitis in the absence of intact NOD1 signaling. Another set of observations pointing to the importance of the role of NOD1 in this chronic pancreatitis model came from studies of BM chimeras, which showed that the development of chronic pancreatitis depended on the expression of NOD1 in the nonhematopoietic cellular compartment. i.e., acinar cells. These studies thus correlated with the observation that stimulation of acinar cells with NOD1 ligand and cerulein led to their production of factors driving the pancreatic inflammation such as CCL2 (as shown in our previous study) ${ }^{15}$ and IL-33 (as shown in the present study). Finally, it should be mentioned that while the administration of NOD1 ligand provided the stimulus for the NOD1 response in this model, under more physiologic conditions, the source of such stimulation is likely to be commensal bacteria that enter the circulation as a result of impaired gut epithelial barrier function.

Type I IFN is another key mediator of the chronic pancreatitis in that mice deficient in the receptor for type I IFN (IFNAR) were unable to develop pancreatitis. BM chimera studies conducted in part with mice lacking IFNAR expression disclosed that both hematopoietic cells and non-hematopoietic cells expressing IFNAR participate in the development of chronic pancreatitis, and thus suggested that type I IFN signaling of both acinar cells and infiltrating macrophages are involved in the pancreatic inflammation. This NOD1-induced type I IFN signals acinar cell induction of Stat3 
and other factors necessary for optimal acinar cell production of CCL2, a chemokine essential to the migration of CCR2 ${ }^{+}$ macrophages into the pancreas. ${ }^{15}$ Its role in macrophage signaling was delineated in studies of other investigators showing that recruitment of Ly6 $\mathrm{C}^{\text {hi }}$ monocytes to a site of inflammation is uniquely dependent on type I IFN induction of monocyte CCR2 expression, the receptor for CCL2. ${ }^{31}$ Thus type I IFN is involved both in the production of CCL2 by acinar cells and in the expression of the CCL2 receptor, CCR2, in macrophages, i.e., events that together facilitate the proinflammatory macrophage infiltration of the pancreas.

Another role of type I IFN in the inflammatory process underlying chronic pancreatitis (in addition to its role in macrophage recruitment described above) relates to the possibility that it induces proinflammatory responses by macrophages that have already entered the pancreas. This possibility arises from a previous study showing that in cerulein-induced pancreatitis myeloid cells recruited into the pancreas produce proinflammatory cytokines such as TNF- $\alpha$, which have the capacity to induce necroptosis in pancreatic acinar cells. ${ }^{32}$ Whether type I IFN is a stimulant of such acinar cell-damaging TNF- $\alpha$ production in the chronic pancreatitis model described here, is suggested by our observation that acinar cells co-cultured with pancreatic $\mathrm{CD} 11 \mathrm{~b}^{+}$cells from mice being subjected to chronic pancreatitis induction, release greatly increased amounts of IL-33 (a possible result of acinar cell necroptosis) and that such IL-33 release is inhibited by both anti-IFNAR $A b$ and anti-TNF- $\alpha$ Ab. These in vitro findings are consistent with the fact that IFNAR-deficient mice exhibit decreased expression of pancreatic TNF- $\alpha$ upon exposure to the FK565-cerulein CP regimen. Thus, type I IFN induces the production of TNF- $\alpha$ by macrophages in this model as shown by Mancuso et al., who reported that type I IFN signaling is required for macrophage production of TNF- $\alpha$ and other proinflammatory cytokines upon stimulation with live bacteria. ${ }^{33}$ Taken together, these data suggest that type I IFN production in chronic pancreatitis is not only responsible for the recruitment of infiltrating macrophages, but also for the stimulation of such macrophages, their production of TNF- $\alpha$ and the subsequent effect of the latter on induction and/or release of IL-33 from damaged pancreatic acinar cells. As such, type I IFN has a multifaceted and central role in the pathogenesis of this model of chronic pancreatitis. Finally, it is important to mention that NOD1 induction of type I IFN due to acinar cell exposure to gut microflora may have effects on a broad range of phagocytic mononuclear cell function. This possibility comes from recent findings showing that gut microflora have been implicated in the activation of phagocytic mononuclear cell activity leading to NK-cell activation. $^{34}$

Yet another essential proinflammatory mediator necessary for the development of chronic pancreatitis in this model is IL-33, a cytokine released by distressed or dying cells such as the acinar cells harboring excessive amounts of trypsin as a result of cerulein administration. The involvement of this cytokine was shown by the fact that mice deficient in either NOD1 or IFNAR did not mount an increased IL-33 response, indicating that abrogation of the pancreatitis prevented the production of this cytokine. More to the point, it was shown by the fact that blockade of IL-33 signaling by administration of an antibody that blocks IL-33 access to its receptor, anti-ST2 Ab, prevents the development of chronic pancreatitis as well as the effects of cytokines induced by IL-33, IL-13, and TGF- $\beta 1$ that are responsible for the fibrosis accompanying chronic pancreatitis. Interestingly, however, the cytokine profile in the pancreatic tissue of mice with the FK565-cerulein chronic pancreatitis model was not a typical IL-33-associated Th2 response accompanied by high levels of IL-4, since in this case, the IL-4 increase was marginal. We attribute this cytokine pattern to the finding that type I IFN induction of Th1 cytokines such as IFN- $\gamma$ and TNF- $\alpha$ were also prominent components of the pancreatic cytokine profile and it is therefore likely that these cytokines were counter-regulating certain aspects of the usual IL-33 response. In any case, the interplay of the IL-33 response and the type I IFN response led to a complex cytokine pattern that contains both Th2 and Th1 elements. One question arising from the presence of both Th1 and Th2 cytokines in this chronic pancreatitis model is the types of immune cells producing IFN- $\gamma$ and IL-13. As mentioned below, we have provided evidence that $\mathrm{CD} 4^{+} \mathrm{T}$ cells are main producers of IL-13. The cells producing IFN- $\gamma$ have not been identified in this study; however, since RAG-1-deficient mice treated with the FK565-cerulein CP regimen exhibited a marked decrease in pancreatic IFN- $\gamma$ expression, we assume that $\mathrm{T}$ cells are main producers of this cytokine. This idea is supported by the finding of Bonilla et al., who showed effective induction of $\mathrm{CD} 8^{+}$ $\mathrm{T}$ cells producing IFN- $\gamma$ by IL-33. ${ }^{35}$

IL-33 is an alarmin-type nuclear cytokine that is released from damaged non-hematopoietic cells. ${ }^{25}$ It is therefore not unexpected that circulating levels of IL-33 are increased in patients with acute pancreatitis, a condition associated with acinar cell disruption. It was nevertheless unclear from prior studies whether the IL-33 produced in pancreatitis has a proinflammatory or anti-inflammatory effect. In one study, focused on mice with acute pancreatitis induced by a cholinedeficient (ethionine-supplemented) diet or high-dose cerulein administration it was found that lack of IL-33 receptor (ST2) expression was associated with more severe inflammation. ${ }^{36}$ Similarly, mice with ST2 deficiency exhibited more severe pancreatitis due to Coxsackievirus B5 infectlon as compared with wild-type mice. ${ }^{37}$ In this case, the more severe disease was attributed to decreased IL-4-mediated M2 macrophage activity leading to decreased regulatory T-cell function; however, IL-33 administration led to decreased viral titer so that its beneficial effect could have been owing to a direct or indirect effect on viral replication. The above studies supporting an antiinflammatory role for IL-33, however, were challenged by Kempuraj et al., who found that acute pancreatitis caused by pancreatic duct ligation was made worse by administration of IL-33 and that the latter was associated with increased NF- $\kappa B$ activation and cytokine/chemokine release. ${ }^{24}$ Similarly, IL-33 was found to have a proinflammatory effect on pancreatic 
carcinoma cells. ${ }^{38}$ The present study, in that it provides unequivocal evidence that IL-33 has a proinflammatory and profibrotic effect in chronic pancreatitis is, at first sight, in apparent conflict with some of the prior studies; however, this apparent conflict may be explained by the fact that the role of IL-33 was examined in experimental pancreatitis induced by different methods than that used here and by the fact that, for the most part, in these studies the effect of IL-33 on fibrosis was not evaluated.

PSCs have been shown to be the major source of extracellular matrix necessary for the development of pancreatic fibrosis. ${ }^{20} \mathrm{~A}$ variety of proinflammatory factors such as IL- 6 , TNF- $\alpha$, and IL-33, all of which are expressed in the mice with chronic pancreatitis due to administration of the FK565-cerulein $\mathrm{CP}$ regimen, have been identified as stimulators of PSC development and function. ${ }^{20,26}$ Type I IFN can be considered an additional factor involved in such development in this model since IFNAR-deficient mice exhibited lack of $\alpha$-SMA expression; however, in this case the effect may be indirect in that type I IFN is acting as an upstream initiator of the inflammatory cascade. In complementary studies we have identified IL-13 as a critical activator of PSCs in this model of chronic pancreatitis. This was evident from studies that showed that neutralization of IL-13 signaling reduced the expression of $\alpha$-SMA. As discussed below, such activating IL-13 originates from $\mathrm{T}$ cells that are part of an adaptive immune response; it is thus clear that while PSC development during chronic pancreatitis depends not only on innate cytokine responses but also on adaptive cytokine responses. Finally, although activated PSCs have been reported to be the source of IL-33 in pancreatic inflammation, ${ }^{26}$ our immunofluorescence studies suggest that IL-33 expressed in the peri-acinar spaces is not co-localized with the marker of PSC, $\alpha$-SMA. Thus, we assume that in this chronic pancreatitis model PSCs are not the main source of IL-33 and this cytokine is more likely to be originating from pancreatic acinar cells.

As shown in recent studies, ILC2s activated by IL-33 have been implicated as a critical player of tissue fibrosis. ${ }^{23,27,28}$ ILC2 contributes to the progression of lung and liver fibrosis by producing a large amount of Th2 cytokines such as IL-5 and IL-13 in response to IL-33..$^{23,27,28}$ Since pancreatic expression of IL-33 and IL-13 is markedly increased in our model of chronic pancreatitis, it was rational to assume that activation of ILC2 producing IL-13 was also involved in the development of chronic pancreatic inflammation and fibrosis. In fact, neutralization of IL-33 or IL-13 signaling pathways protects mice from chronic pancreatic inflammation and fibrosis in our model. It should be noted, however, that RAG1-deficient mice are completely protected from chronic pancreatitis with diminished pancreatic expression of IL-13, whereas expression of innate immune mediators such as IFN- $\beta$, IL-33, and CCL2 were preserved. In addition, we found pancreatic $\mathrm{CD} 4^{+} \mathrm{T}$ cells isolated from mice treated with the FK565-cerulein CP regimen produced a large amount of IL-13 upon stimulation with anti-CD3 Ab and CD28 Ab. On the basis of these data, we speculate that $\mathrm{T}$ cells rather than ILC2s are major source of IL-13 in this model of chronic pancreatitis and that chronic fibroinflammatory responses are mediated by classical Th2 cells. ${ }^{39}$ Confirmation of this idea awaits further study addressing phenotypic analysis of ILC2 and Th2 cells in this model and we cannot exclude the possibility that collaborative interactions between ILC2s and Th2 cells exacerbate chronic fibroinflammatory responses of the pancreas as suggested by a recent study. ${ }^{40}$

In conclusion, we have established a unique model of chronic pancreatitis induced by repetitive administration of low-dose cerulein and NOD1 ligand. This model exhibits key characteristics of chronic pancreatitis occurring in human disease, namely, pancreatic atrophy and fibrosis and establishes that these characteristics are caused by the production of IL-33 and its downstream product, IL-13. The fact that activation of NOD1 has a central role in this model suggests the possibility that human chronic pancreatitis is caused by initial intrapancreatic events that lead to the entry of commensal organisms into the pancreas and the subsequent stimulation of proinflammatory innate and adaptive immune mechanisms that sustain prolonged pancreatitis. However, studies in humans with pancreatitis will be necessary to explore this possibility.

\section{METHODS}

Mice. C57BL/6 mice were purchased from Japan SLC (Hamamatsu, Japan). NOD1-deficient mice, IFNAR-deficient mice, and GFPtransgenic mice were used as described previously. ${ }^{21}$ RAG1-deficient mice were kindly provided by Dr K Suzuki (Kyoto University Graduate School of Medicine). Mice were reared under specific pathogen free conditions. Animal use adhered to the Kyoto University animal-care guidelines, and protocols of animal experiments were approved by the review boards of Kyoto University.

Induction of pancreatitis. Mice received IP injection of FK565 (50 $\mu \mathrm{g}$, Astellas Pharma, Tokyo, Japan) in combination with IP injection of cerulein $\left(20 \mu \mathrm{g} \mathrm{kg}^{-1}\right.$, Sigma, St Louis, MO). Mice received each treatment regimen twice a week for a total of 14 times and then sera and pancreas tissue were obtained. In some experiments, mice were treated with anti-ST2 Ab $(100 \mu \mathrm{g}$ per mouse, R\&D systems, Minneapolis, $\mathrm{MN})$, anti-IL-13 Ab (100 $\mu$ g per mouse, eBioscience, San Diego, CA) or rat IgG (100 $\mu \mathrm{g}$ per mouse, Sigma). In some experiments, mice received four hourly IP injection of cerulein $\left(100 \mu \mathrm{g} \mathrm{kg}^{-1}\right)$ twice a week for a total of 14 times. Serum levels of amylase were determined by the biochemical analyzer, SPOTCHEM (Arkray, Kyoto, Japan). Pancreatic lysate were prepared as described previously. ${ }^{15}$ Pancreatic levels of hydroxyproline were determined by the hydroxyproline assay kit (QuickZyme Biosciences, Leiden, The Netherlands).

Bone marrow transplantation. For the generation of BM-chimeric mice, recipient mice were irradiated 10 Gray and reconstituted with BM cells $\left(2 \times 10^{6}\right.$ per each recipient mouse $)$ from the donor mice via the tail vein injection. Mice were used for the induction of experimental pancreatitis at 6-8 weeks after the BM transplantation.

Enzyme-linked immunosorbent assay. Protein concentrations of cytokines and chemokines were determined by eBioscience ELISA kits for mouse IL-6, CCL2, TNF- $\alpha$, IFN- $\gamma$, IL-33, and IL-13. R\&D systems ELISA kits were used for the measurement of mouse IFN- $\beta$, CXCL9, and CXCL10. Concentration of TGF- $\beta 1$ was determined by Promega ELISA kit (Madison, WI).

Immunofluoresence and immunohistochemical analysis. Pancreas tissues were harvested and fixed in $10 \%$ formalin. Deparaffinized 
sections were incubated with anti-IL-33 Ab (Abcam, Cambridge, MA), anti-SMA Ab (Abcam), ${ }^{41,42}$ anti-fibronectin $\mathrm{Ab}$ (Abcam), ${ }^{43}$ anti-CD3 $\mathrm{Ab}$ (Abcam), ${ }^{44}$ and anti-CD $11 \mathrm{~b}$ Ab (Abcam). ${ }^{45}$ Protein expression was visualized by the Dako Envison + system (DAKO JAPAN, Tokyo, Japan). For the immunofluorescence analysis, deparaffinized sections were incubated with mouse anti-IL-33 Ab (Abcam), rabbit anti-SMA Ab (Abcam), mouse anti-SMA Ab (Abcam), mouse anti-pI B $\alpha$ (CST, Cambridge, MA), ${ }^{15}$ rabbit anti-amylase (Sigma), ${ }^{15}$ rabbit anti-GFP (CST), or mouse anti-pStat $3(\mathrm{CST})^{15}$ followed by the incubation with Alexa 488 or Alexa 546-conjugated anti-mouse or rabbit IgG (Invitrogen, Carlsbad, CA). Sirius Red staining was performed by using Picosirius red stain kit (Polysciences Inc., Warrington, PA). At least two immunohistochemical and immunofluorescence photographs were taken by microscopy (Biozero BZ-8100, Keyence, Osaka, Japan) from each slide prepared from mice treated with cerulein and/or FK565. The degree of pancreatic infiltration of PSCs was determined by counting the number of $\alpha$-SMA ${ }^{+}$cells in high-power fields in each slide. Pathological scores of chronic pancreatitis was determined as previously described with some modifications. ${ }^{46}$ Within pancreatic sections, areas of abnormal pancreatic tissue architecture were graded as follows: $0=$ absent, $1=$ rare, $2=\operatorname{minimal}<10 \%, 3=$ moderate $10-50 \%$, and $4=$ severe $>50 \%$. Within these areas, glandular atrophy were graded as follows: $0=$ absent, $1=$ minimal $<10 \%, 2=$ moderate $10-50 \%$, and $3=$ severe $>50 \%$. In addition, the presence of immune cells was graded as: $0=$ absent, $1=$ minimal, $2=$ mild, $3=$ moderate, and $4=$ severe. The total histological score is given as the pancreatic tissue architecture plus the glandular atrophy plus the presence of immune cells.

Isolation of pancreatic acinar cells and immune cells. Pancreatic acinar cells $\left(5 \times 10^{5}\right.$ per $\left.\mathrm{ml}\right)$ were isolated as described previously ${ }^{47}$ and stimulated with cerulein $\left(10^{-10} \mathrm{M}\right)$ and FK565 $\left(100 \mathrm{ng} \mathrm{ml}^{-1}\right)$ for $24 \mathrm{~h}$. Pancreatic immune cells were isolated as described previously. ${ }^{48}$ Pancreatic immune cells were stained with $\mathrm{PE}$ or FITC-conjugated Gr-1 (Miltenyibiotec, Auburn, CA), F4-80 (Biolegend, San Diego, CA), CD11b (eBioscience), or CD11c Ab (eBioScience) for the flow-cytometric analysis (Accuri C6 cytometer, $\mathrm{BD}$ Bioscience, San Jose, CA). CD4 ${ }^{+}$cells or $\mathrm{CD} 1 \mathrm{~b}^{+}$cells were isolated from pancreatic immune cells by using CD4 or CD11b microbeads (Mitenyibiotec). The purity of $\mathrm{CD}^{+}$cells or $\mathrm{CD} 11 \mathrm{~b}^{+}$ cells was more than $80 \%$ as assessed by flow-cytometric analysis. Pancreatic immune cells were stimulated with anti-CD3 Ab $\left(5 \mu \mathrm{g} \mathrm{ml}^{-1}\right.$, eBioscinece) and anti-CD28 $\mathrm{Ab}\left(10 \mu \mathrm{g} \mathrm{ml}^{-1}, \mathrm{BD}\right.$ Bioscience) for $48 \mathrm{~h}$ to determine the production of IL-13. In the coculture experiments composed of pancreatic acinar cells $\left(5 \times 10^{5}\right.$ per $\mathrm{ml})$ and CD $11 \mathrm{~b}^{+}$cells $\left(5 \times 10^{5}\right.$ per ml), neutralizing Ab against TNF- $\alpha$ (R\&D systems) or IFNAR (BD Bioscience) was added at the concentration of $50 \mu \mathrm{g} \mathrm{ml}^{-1}$.

Statistical analysis. Student's $t$ test was used to evaluate the significance of the differences. Statistical analysis was performed with the Prism (Graphpad, software, La Jolla, CA). A value of $P<0.05$ was regarded as statistically significant.

SUPPLEMENTARY MATERIAL is linked to the online version of the paper at http://www.nature.com/mi

\section{ACKNOWLEDGMENTS}

This work was supported by Grant-in-Aid for Scientific Research (25293172, 15K15370) from the Japan Society for the Promotion of Science, the Japanese Society of Gastroenterology, the Kato Memorial Trust for Nambyo Research, and the Special Coordination Funds by the Ministry of Education, Culture, Sports, Science and Technology of Japan and Astellas Pharma. in Creation of Innovation Centers for Advanced Interdisciplinary Research Areas Program, and the Health and Labor Sciences Research Grants for Research on Intractable Diseases from the Ministry of Health, Labor and Welfare, Japan.

\section{AUTHOR CONTRIBUTIONS}

Study concept and design; TW, WS. Acquisition of data; TW, YS, NY, HE. Analysis and interpretation of data; TW, WS. Drafting of the manuscript; TW, TS, WS. Study supervision; MK, TC, WS.

\section{DISCLOSURE}

The authors declare no conflict of interest.

c) 2016 Society for Mucosal Immunology

\section{REFERENCES}

1. Frossard, J.L., Steer, M.L. \& Pastor, C.M. Acute pancreatitis. Lancet 371, 143-152 (2008).

2. Braganza, J.M., Lee, S.H., McCloy, R.F. \& McMahon, M.J. Chronic pancreatitis. Lancet 377, 1184-1197 (2011).

3. Steer, M.L., Waxman, I. \& Freedman, S. Chronic pancreatitis. N. Engl. J. Med. 332, 1482-1490 (1995).

4. Sah, R.P. \& Saluja, A. Molecular mechanisms of pancreatic injury. Curr. Opin. Gastroenterol. 27, 444-451 (2011).

5. Saluja, A.K., Lerch, M.M., Phillips, P.A. \& Dudeja, V. Why does pancreatic overstimulation cause pancreatitis?. Annu. Rev. Physiol. 69, 249-269 (2007).

6. Whitcomb, D.C. Genetic aspects of pancreatitis. Annu. Rev. Med. 61, 413-424 (2010).

7. Etemad, B. \& Whitcomb, D.C. Chronic pancreatitis: diagnosis, classification, and new genetic developments. Gastroenterology 120, 682-707 (2001).

8. Dawra, R. et al. Intra-acinar trypsinogen activation mediates early stages of pancreatic injury but not inflammation in mice with acute pancreatitis. Gastroenterology 141, 2210-2217 e2212 (2011).

9. Sah, R.P., Dudeja, V., Dawra, R.K. \& Saluja, A.K. Cerulein-induced chronic pancreatitis does not require intra-acinar activation of trypsinogen in mice. Gastroenterology 144, 1076-1085 e1072 (2013).

10. Yamaguchi, H., Kimura, T., Mimura, K. \& Nawata, H. Activation of proteases in cerulein-induced pancreatitis. Pancreas 4, 565-571 (1989).

11. Ji, B. \& Logsdon, C.D. Digesting new information about the role of trypsin in pancreatitis. Gastroenterology 141, 1972-1975 (2011).

12. Akira, S. \& Takeda, K. Toll-like receptor signalling. Nat. Rev. Immunol. 4, 499-511 (2004).

13. Strober, W., Murray, P.J., Kitani, A. \& Watanabe, T. Signalling pathways and molecular interactions of NOD1 and NOD2. Nat. Rev. Immunol. 6, 9-20 (2006).

14. Kono, H. \& Rock, K.L. How dying cells alert the immune system to danger. Nat. Rev. Immunol. 8, 279-289 (2008).

15. Tsuji, Y., Watanabe, T., Kudo, M., Arai, H., Strober, W. \& Chiba, T. Sensing of commensal organisms by the intracellular sensor NOD1 mediates experimental pancreatitis. Immunity 37, 326-338 (2012).

16. Mithofer, K., Fernandez-del Castillo, C., Ferraro, M.J., Lewandrowski, K., Rattner, D.W. \& Warshaw, A.L. Antibiotic treatment improves survival in experimental acute necrotizing pancreatitis. Gastroenterology 110 , 232-240 (1996)

17. Hoque, R., Malik, A.F., Gorelick, F. \& Mehal, W.Z. Sterile inflammatory response in acute pancreatitis. Pancreas 41, 353-357 (2012).

18. Hoque, R. et al. TLR9 and the NLRP3 inflammasome link acinar cell death with inflammation in acute pancreatitis. Gastroenterology 141, 358-369 (2011).

19. Ulmasov, B., Oshima, K., Rodriguez, M.G., Cox, R.D. \& NeuschwanderTetri, B.A. Differences in the degree of cerulein-induced chronic pancreatitis in C57BL/6 mouse substrains lead to new insights in identification of potential risk factors in the development of chronic pancreatitis. Am. J. Pathol. 183, 692-708 (2013).

20. Masamune, A. \& Shimosegawa, T. Pancreatic stellate cells-multi-functional cells in the pancreas. Pancreatology 13, 102-105 (2013).

21. Watanabe, T. et al. NOD1 contributes to mouse host defense against Helicobacter pylori via induction of type I IFN and activation of the ISGF3 signaling pathway. J. Clin. Invest. 120, 1645-1662 (2010). 
22. Fichtner-Feigl, S., Strober, W., Geissler, E.K. \& Schlitt, H.J. Cytokines mediating the induction of chronic colitis and colitis-associated fibrosis. Mucosal Immunol. 1 Suppl 1, S24-S27 (2008).

23. McHedlidze, T. et al. Interleukin-33-dependent innate lymphoid cells mediate hepatic fibrosis. Immunity 39, 357-371 (2013).

24. Kempuraj, D., Twait, E.C., Williard, D.E., Yuan, Z., Meyerholz, D.K. \& Samuel, I. The novel cytokine interleukin-33 activates acinar cell proinflammatory pathways and induces acute pancreatic inflammation in mice. PLOS ONE 8, e56866 (2013).

25. Cayrol, C. \& Girard, J.P. IL-33: an alarmin cytokine with crucial roles in innate immunity, inflammation and allergy. Curr. Opin. Immunol. 31C, 31-37 (2014).

26. Masamune, A., Watanabe, T., Kikuta, K., Satoh, K., Kanno, A. \& Shimosegawa, T. Nuclear expression of interleukin-33 in pancreatic stellate cells. Am. J. Physiol. Gastrointest. Liver Physiol. 299, G821-G832 (2010).

27. Li, D. et al. IL-33 promotes ST2-dependent lung fibrosis by the induction of alternatively activated macrophages and innate lymphoid cells in mice. J. Allergy Clin. Immunol. 134, 1422-1432 e1411 (2014).

28. Seidelin, J.B., Rogler, G. \& Nielsen, O.H. A role for interleukin-33 in $T(H) 2-$ polarized intestinal inflammation?. Mucosal Immunol. 4, 496-502 (2011).

29. Monticelli, L.A. et al. Innate lymphoid cells promote lung-tissue homeostasis after infection with influenza virus. Nat. Immunol. 12, 1045-1054 (2011).

30. Li, J. et al. Biliary repair and carcinogenesis are mediated by IL-33-dependent cholangiocyte proliferation. J. Clin. Invest. 124, 3241-3251 (2014).

31. Lee, P.Y. et al. Type I interferon modulates monocyte recruitment and maturation in chronic inflammation. Am. J. Pathol. 175, 2023-2033 (2009).

32. He, S. et al. Receptor interacting protein kinase-3 determines cellular necrotic response to TNF-alpha. Cell 137, 1100-1111 (2009).

33. Mancuso, G. et al. Type I IFN signaling is crucial for host resistance against different species of pathogenic bacteria. J. Immunol. 178, 3126-3133 (2007).

34. Ganal, S.C. et al. Priming of natural killer cells by nonmucosal mononuclear phagocytes requires instructive signals from commensal microbiota. Immunity 37, 171-186 (2012).

35. Bonilla, W.V. et al. The alarmin interleukin-33 drives protective antiviral CD8(+) T cell responses. Science 335, 984-989 (2012).
36. Ouziel, R. et al. The ST2 pathway is involved in acute pancreatitis: a translational study in humans and mice. Am. J. Pathol. 180, 2330-2339 (2012).

37. Sesti-Costa, R. et al. The IL-33/ST2 pathway controls coxsackievirus B5-induced experimental pancreatitis. J. Immunol. 191, 283-292 (2013).

38. Schmieder, A., Multhoff, G. \& Radons, J. Interleukin-33 acts as a pro-inflammatory cytokine and modulates its receptor gene expression in highly metastatic human pancreatic carcinoma cells. Cytokine 60, 514-521 (2012).

39. Ochi, A. et al. MyD88 inhibition amplifies dendritic cell capacity to promote pancreatic carcinogenesis via Th2 cells. J. Exp. Med. 209, 1671-1687 (2012).

40. Liu, B., Lee, J.B., Chen, C.Y., Hershey, G.K. \& Wang, Y.H. Collaborative interactions between type 2 innate lymphoid cells and antigen-specific CD4 + Th2 cells exacerbate murine allergic airway diseases with prominent eosinophilia. J. Immunol. 194, 3583-3593 (2015).

41. Zhang, S.M. et al. Interferon regulatory factor 8 modulates phenotypic switching of smooth muscle cells by regulating the activity of myocardin. Mol. Cell Biol. 34, 400-414 (2014).

42. Hagiwara, K. et al. Molecular and cellular features of murine craniofacial and trunk neural crest cells as stem cell-like cells. PLOS ONE 9, e84072 (2014).

43. Conway, M.J. et al. Mosquito saliva serine protease enhances dissemination of dengue virus into the mammalian host. J. Virol. 88, 164-175 (2014).

44. Simons, B.W. et al. A human prostatic bacterial isolate alters the prostatic microenvironment and accelerates prostate cancer progression. J. Pathol. 235, 478-489 (2015).

45. Rommer, A. et al. EVl1 inhibits apoptosis induced by antileukemic drugs via upregulation of CDKN1A/p21/WAF in human myeloid cells. PLOS ONE 8, e56308 (2013).

46. van Westerloo, D.J. et al. Therapeutic effects of troglitazone in experimental chronic pancreatitis in mice. Am. J. Pathol. 166, 721-728 (2005).

47. Gout, J. et al. Isolation and culture of mouse primary pancreatic acinar cells. J. Vis. Exp. 78, e50514 (2013).

48. Arai, Y. et al. Plasmacytoid dendritic cell activation and IFN-alpha production are prominent features of murine autoimmune pancreatitis and human igg4-related autoimmune pancreatitis. J. Immunol. 195, 3033-3044 (2015). 